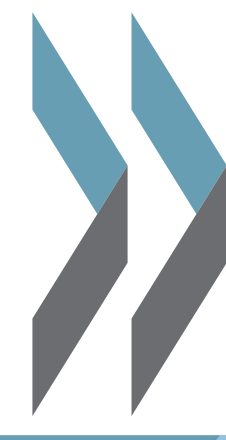

Les principes directeurs de l'OCDE à l'intention des entreprises multinationales

\title{
ÉDITION 2011
}





\section{Les principes directeurs de l'OCDE à l'intention des entreprises multinationales}

\section{Édition 2011}


Cet ouvrage est publié sous la responsabilité du Secrétaire général de l'OCDE. Les opinions et les interprétations exprimées ne reflètent pas nécessairement les vues de l'OCDE ou des gouvernements de ses pays membres.

\section{Merci de citer cet ouvrage comme suit :}

OCDE (2011), Les principes directeurs de l'OCDE à l'intention des entreprises multinationales, Éditions OCDE.

http://dx.doi.org/10.1787/9789264115439-fr

ISBN 978-92-64-11542-2 (imprimé)

ISBN 978-92-64-11543-9 (PDF)

Les corrigenda des publications de l'OCDE sont disponibles sur : www.oecd.org/editions/corrigenda.

(c) OCDE 2011

Vous êtes autorisés à copier, télécharger ou imprimer du contenu OCDE pour votre utilisation personnelle. Vous pouvez inclure des extraits des publications, des bases de données et produits multimédia de l'OCDE dans vos documents, présentations, blogs, sites Internet et matériel d'enseignement, sous réserve de faire mention de la source OCDE et du copyright. Les demandes pour usage public ou commercial ou de traduction devront être adressées à rights@oecd.org. Les demandes d'autorisation de photocopier une partie de ce contenu à des fins publiques ou commerciales peuvent être obtenues auprès du Copyright Clearance Center (CCC) info@copyright.com ou du Centre français d'exploitation du droit de copie (CFC) contact@cfcopies.com. 


\section{Avant-propos}

Les Principes directeurs de l'OCDE à l'intention des entreprises multinationales sont des recommandations que les gouvernements adressent aux entreprises multinationales exerçant leurs activités dans les pays adhérents ou à partir de ces derniers. Ils contiennent des principes et des normes non contraignants destinés à favoriser une conduite raisonnable des entreprises dans un environnement mondialisé, en conformité avec les législations applicables et les normes internationalement admises. Les Principes directeurs constituent, en matière de conduite responsable des entreprises, le seul code exhaustif convenu à l'échelon multilatéral que les gouvernements se sont engagés à promouvoir.

Les recommandations énoncées dans les Principes directeurs expriment les valeurs partagées par les gouvernements des pays dont provient une grande partie de l'investissement direct international et dans lesquels sont implantées nombre des entreprises multinationales parmi les plus grandes. Les Principes directeurs ont vocation à promouvoir une contribution positive des entreprises au progrès économique, environnemental et social partout dans le monde.

Les Principes directeurs sont étayés par un mécanisme de mise en œuvre unique en son genre, les Points de contact nationaux (PCN), qui sont les instances établies par les gouvernements adhérents pour promouvoir et mettre en œuvre les Principes directeurs. Les PCN aident les entreprises et leurs parties prenantes à prendre les mesures adéquates pour en renforcer encore l'application. Ils constituent également un pôle de médiation et de conciliation permettant de résoudre les problèmes pratiques susceptibles de se poser.

Le 4 mai 2010, les gouvernements de 42 pays membres et non membres de l'OCDE ayant adhéré à la Déclaration de l'OCDE sur l'investissement international et les entreprises multinationales et à la Décision connexe ont lancé les travaux de mise à jour des Principes directeurs afin de tenir compte des évolutions du paysage de l'investissement international et des entreprises multinationales intervenues depuis la dernière révision effectuée en 2000. Les modifications convenues dans ce cadre ont pour objet de 
garantir que les Principes directeurs continueront à jouer leur rôle d'instrument international de premier plan dans la promotion d'une conduite responsable des entreprises.

Les Principes directeurs mis à jour et la Décision connexe ont été adoptés par les 42 pays adhérents le 25 mai 2011 lors de la Réunion ministérielle du $50^{\text {ème }}$ anniversaire de l'OCDE.

Parmi les modifications apportées aux Principes directeurs, on peut citer :

- Un nouveau chapitre sur les droits de l'homme inspiré des Principes directeurs pour les entreprises et les droits de l'homme : mise en œuvre du cadre «Protéger, respecter et réparer» établi par les Nations Unies.

- Une approche nouvelle et plus complète de la diligence et de la gestion responsable de la chaîne d'approvisionnement qui représente un progrès significatif par rapport aux conceptions antérieures.

- Des changements importants dans de nombreux chapitres spécialisés concernant par exemple l'emploi et les relations sociales, la lutte contre la corruption, la sollicitation de pots-de-vin et d'autres formes d'extorsion, l'environnement, les intérêts des consommateurs, la publication d'informations ou encore la fiscalité.

- Des lignes directrices de procédure plus claires et plus strictes destinées à renforcer le rôle des $\mathrm{PCN}$, à en améliorer les performances et à favoriser l'équivalence fonctionnelle.

- Un agenda proactif de mise en œuvre destiné à aider les entreprises à assumer leurs responsabilités au fur et à mesure que de nouveaux défis se présenteront à elles.

La mise à jour des Principes directeurs a été menée par les gouvernements adhérents qui ont procédé à des consultations intensives avec un large éventail de parties prenantes et de partenaires. Tous les pays du G20 non adhérents ont été invités à y prendre part sur un pied d'égalité ; ils ont apporté à ce titre des contributions importantes, de même que les participants aux consultations régionales organisées en Asie, en Afrique, en Amérique latine ainsi qu'au Moyen-Orient et en Afrique du Nord. Le Comité consultatif économique et industriel auprès de l'OCDE (BIAC), la Commission syndicale consultative auprès de l'OCDE (TUAC) et OECD Watch se sont fait les porte-paroles des entreprises, des organisations syndicales et des organisations non gouvernementales (ONG) grâce à des consultations régulières et à leur participation active au groupe consultatif du Président du Groupe de travail chargé de la mise à jour des Principes 
directeurs. Le Représentant spécial pour la question des droits de l'homme et des sociétés transnationales et autres entreprises auprès du Secrétaire général des Nations Unies, le Professeur John Ruggie, l'Organisation internationale $\mathrm{du}$ travail et d'autres organisations internationales ont également apporté de larges contributions à la mise à jour.

Au sein de l'OCDE, le Comité des affaires fiscales; le Comité de la concurrence; le Comité de l'emploi, du travail et des affaires sociales; le Comité sur la gouvernance d'entreprise ; le Comité de la politique à l'égard des consommateurs; le Comité des politiques d'environnement; et le Groupe de travail sur la corruption dans le cadre de transactions commerciales internationales ont pour leur part contribué à la révision des différents chapitres spécialisés des Principes directeurs relevant de leurs activités.

Les travaux consacrés à la mise à jour ont été menés avec le soutien de la Division de l'investissement qui a assuré le Secrétariat du Comité de l'investissement de l'OCDE, en collaboration avec la Direction des affaires juridiques, le Centre de politique et d'administration fiscales, la Division de lutte contre la corruption, la Division de la concurrence, la Division des affaires d'entreprise, la Division de l'analyse et des politiques de l'emploi, la Division de l'intégration de l'environnement et de l'économie et la Division de la politique de l'information, des communications et des consommateurs. 



\section{Table des matières}

\section{La Déclaration sur l'investissement international}

et les entreprises multinationales

Partie I

Les Principes directeurs de l'OCDE à l'intention des entreprises multinationales

Recommandations pour une conduite responsable

des entreprises dans le contexte international

\begin{tabular}{|c|c|c|}
\hline réfa & & \\
\hline I & Concepts et principes. & \\
\hline II. & Principes généraux.. & \\
\hline III. & Publication d'informations & \\
\hline IV. & Droits de l'homme. & \\
\hline $\mathrm{V}$. & Emploi et relations professionnelles...... & \\
\hline VI. & Environnement & \\
\hline & $\begin{array}{l}\text { Lutte contre la corruption, la sollicitation de pots-de-vin et d'autres } \\
\text { formes d'extorsion. }\end{array}$ & \\
\hline VIII. & Intérêts des consommateurs. & 6 \\
\hline IX. & Science et technologie & 6 \\
\hline $\mathrm{X}$. & Concurrence. & 6 \\
\hline II. & & \\
\hline
\end{tabular}

\begin{tabular}{|c|}
\hline Partie II \\
$\begin{array}{c}\text { Procédures de mise en ouvre des Principes directeurs de l'OCDE } \\
\text { à l'intention des entreprises multinationales }\end{array}$ \\
\hline
\end{tabular}

Amendement de la décision du Conseil sur les principes directeurs

de l'OCDE a l'intention des entreprises multinationales

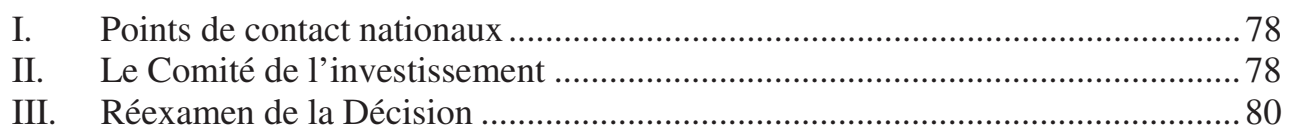




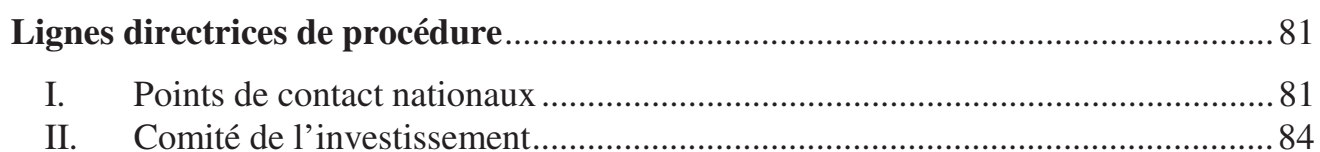

Commentaire sur les procédures de mise en ouvre des Principes directeurs

de l'OCDE à l'intention des entreprises multinationales

I. Commentaire sur les lignes directrices de procédure pour les Points de contact nationaux

II. Commentaire sur les lignes directrices de procédure pour le Comité de l'investissement 


\title{
LA DÉCLARATION SUR L'INVESTISSEMENT INTERNATIONAL ET LES ENTREPRISES MULTINATIONALES
}

\author{
25 mai 2011 \\ LES GOUVERNEMENTS ADHÉRENTS ${ }^{1}$
}

CONSIDÉRANT :

Que les investissements internationaux revêtent une importance majeure pour l'économie mondiale et ont contribué dans une mesure considérable au développement de leur pays.

Que les entreprises multinationales jouent un rôle important dans le processus d'investissement.

Que la coopération internationale peut améliorer le climat des investissements étrangers, favoriser la contribution positive que les entreprises multinationales peuvent apporter au progrès économique, social et environnemental, et permettre de réduire au minimum et de résoudre les difficultés qui peuvent résulter de leurs opérations.

Que les avantages de la coopération internationale peuvent être accrus en abordant les questions afférentes à l'investissement international et aux entreprises multinationales au moyen d'un ensemble équilibré d'instruments interdépendants. Membres de l'OCDE ainsi que l'Argentine, le Brésil, l'Égypte, la Lettonie, la Lituanie, le Maroc, le Pérou et la Roumanie. La Communauté européenne est invitée à s'associer à la section sur le Traitement national pour les questions relevant de sa compétence. 


\section{DÉCLARENT:}

Principes

directeurs à

l'intention des

entreprises

multinationales

I. Qu'ils recommandent conjointement aux entreprises multinationales opérant sur le territoire de leurs pays ou à partir de celui-ci d'observer les Principes directeurs énoncés dans l'Annexe 1 ci-jointe ${ }^{2}$, en tenant compte des considérations et des précisions qui figurent dans la Préface et le premier chapitre desdits Principes et qui en font partie intégrante ;

Traitement national

II. 1. Que les gouvernements adhérents, compte tenu de la nécessité de maintenir l'ordre public, de protéger les intérêts essentiels de leur sécurité et de remplir leurs engagements concernant la paix et la sécurité internationales, devraient accorder aux entreprises opérant sur leur territoire et qui appartiennent à, ou sont contrôlées directement ou indirectement par des ressortissants d'un autre gouvernement adhérent (appelés ci-dessous «entreprises sous contrôle étranger »), un régime résultant de leurs lois, réglementations et pratiques administratives (appelé ci-dessous « Traitement national ») qui, en harmonie avec le droit international, ne sera pas moins favorable que celui dont bénéficient dans les mêmes circonstances les entreprises nationales ;

2. Que les gouvernements adhérents envisageront d'appliquer le «Traitement national » à l'égard d'autres pays que les gouvernements adhérents ;

3. Que les gouvernements adhérents s'efforceront de veiller à ce que les subdivisions territoriales appliquent le « Traitement national »;

4. Que la Déclaration ne concerne pas le droit de tout gouvernement adhérent de réglementer l'admission des investissements étrangers ou les conditions d'établissement des entreprises étrangères. 


\begin{abstract}
Obligations III. Qu'ils coopéreront afin d'éviter ou d'atténuer contradictoires l'imposition d'obligations contradictoires aux entreprises multinationales et qu'ils prendront en compte les considérations générales et les modalités pratiques qui sont énoncées à l'annexe 2 ci-après ${ }^{3}$.
\end{abstract}

Stimulants et IV. 1. Qu'ils reconnaissent la nécessité de renforcer leur obstacles à l'investissement international

Procédures de V. consultation coopération dans 1e domaine des investissements directs internationaux ;

2. Qu'ils reconnaissent ainsi la nécessité de tenir dûment compte des intérêts des gouvernements adhérents qui seraient affectés par les lois, réglementations et pratiques administratives (appelées ci-dessous «mesures ») visant spécifiquement les investissements directs internationaux et constituant des stimulants ou des obstacles à ces investissements ;

3. Que les gouvernements adhérents s'efforceront de rendre ces mesures aussi transparentes que possible, de façon que leur importance et leur objet puissent être facilement déterminés et que des informations à leur sujet puissent être facilement obtenues ;

Qu'ils sont prêts à se consulter sur les questions ci-dessus, en application des Décisions pertinentes du Conseil ;

Réexamen VI. Qu'ils réexamineront les questions ci-dessus périodiquement, en vue de rendre plus efficace la coopération économique internationale entre gouvernements adhérents dans les domaines relatifs à l'investissement international et aux entreprises multinationales.

3. Le texte des considérations générales et approches pratiques, relatif aux obligations contradictoires imposées aux entreprises multinationales est disponible sur le site Web de l'OCDE www.oecd.org/daf/investissement/. 

Partie I

\section{Les Principes directeurs de l'OCDE à l'intention des entreprises multinationales}

\section{Recommandations pour une conduite responsable des entreprises dans le contexte international}

\section{Texte et commentaires}

Note du Secrétariat : Les commentaires ont été élaborés par le Comité de l'investissement en session élargie, y compris les huit pays non-Membres* adhérant à la Déclaration de l'investissement international et les entreprises internationales, afin de fournir des informations et des explications sur le texte des Principes directeurs de l'OCDE à l'intention des entreprises multinationales et la Décision du Conseil sur les Principes directeurs de l'OCDE à l'intention des entreprises multinationales. Ils ne font partie de la Déclaration sur l'investissement international et les entreprises multinationales ni à la Décision du Conseil sur les Principes directeurs de l'OCDE à l'intention des entreprises multinationales.

Dans cette publication, les commentaires sont situés après le chapitre auquel ils font référence et sont numérotés consécutivement de 1 à 106. Argentina, Brésil, Égypte, Lettonie, Lituanie, Maroc, Pérou et Romanie. 



\section{Préface}

Les Principes directeurs de l'OCDE à l'intention des entreprises multinationales (les Principes directeurs) sont des recommandations que les gouvernements adressent aux entreprises multinationales. Les Principes directeurs visent à faire en sorte que les activités des entreprises multinationales s'exercent en harmonie avec les politiques des gouvernements, à renforcer la confiance mutuelle entre les entreprises et les sociétés dans lesquelles elles exercent leurs activités, à améliorer l'environnement pour l'investissement étranger et à accroître la contribution des entreprises multinationales au développement durable. Les Principes directeurs font partie de la Déclaration de l'OCDE sur l'investissement international et les entreprises multinationales, dont les autres éléments concernent le traitement national, les obligations contradictoires imposées aux entreprises ainsi que les incitations et les obstacles à l'investissement international. Les Principes directeurs énoncent des principes et des normes volontaires de conduite responsable des entreprises, en conformité avec les législations applicables et les normes internationalement admises. Toutefois, les pays adhérant aux Principes directeurs prennent l'engagement contraignant de les mettre en œuvre conformément à la Décision du Conseil relative aux Principes directeurs de l'OCDE à l'intention des entreprises multinationales. En outre, les sujets abordés dans les Principes directeurs peuvent également faire l'objet de législations nationales et d'engagements internationaux.

Les activités internationales des entreprises ont subi de profonds changements structurels et les Principes directeurs ont eux-mêmes évolué pour tenir compte de ces changements. Avec le développement des secteurs des services et des secteurs à forte intensité de savoir et l'expansion de l'économie de l'Internet, les entreprises opérant dans les services et la technologie jouent un rôle de plus en plus important sur le marché international. Les grandes entreprises représentent toujours une forte proportion de l'investissement international et les grandes fusions internationales se multiplient. Dans le même temps, les petites et moyennes entreprises investissent elles aussi davantage à l'étranger et jouent maintenant un rôle non négligeable sur la scène internationale. Comme leurs homologues nationales, les entreprises multinationales ont évolué et font 
aujourd'hui appel à un plus large éventail de mécanismes industriels et commerciaux et de formes d'organisation. La conclusion d'alliances stratégiques et le resserrement des liens avec les fournisseurs et les soustraitants ont tendance à brouiller les frontières de l'entreprise.

La rapide évolution de la structure des entreprises multinationales se reflète aussi dans leurs activités dans le monde en développement, où l'investissement direct étranger croît rapidement. Dans les pays en développement, les entreprises multinationales ont diversifié leurs activités, autrefois limitées à la production primaire et aux industries extractives, et se sont lancées dans la fabrication et le montage, la mise en valeur du marché intérieur et les services. L'apparition d'entreprises multinationales implantées dans des pays en développement s'imposant comme des investisseurs internationaux de poids constitue un autre fait marquant.

Par le biais des échanges et des investissements internationaux, les activités des entreprises multinationales ont permis de renforcer et d'approfondir les liens entre les différents pays et les différentes régions du monde. De telles activités sont très bénéfiques pour les pays d'origine comme pour les pays d'accueil. Les avantages qui en découlent se concrétisent lorsque les entreprises multinationales fournissent à des prix concurrentiels les produits ou services recherchés par les consommateurs et lorsqu'elles assurent un juste rendement à ceux qui leur apportent des capitaux. En outre, les activités des entreprises multinationales dans le domaine des échanges et de l'investissement contribuent à l'utilisation efficace du capital, de la technologie et des ressources humaines et naturelles. Elles facilitent les transferts de technologie entre les différentes régions du monde et la mise au point de technologies adaptées aux conditions locales. Grâce à une formation institutionnalisée et à l'apprentissage en cours d'emploi, les entreprises contribuent également à valoriser le capital humain et à créer des perspectives d'emploi dans les pays d'accueil.

La nature, l'étendue et la rapidité des changements économiques représentent de nouveaux défis stratégiques pour les entreprises et leurs parties prenantes. Les entreprises multinationales ont ainsi l'occasion de mettre en place des politiques de pratiques exemplaires dans le domaine du développement durable qui visent à la cohérence des objectifs économiques, environnementaux et sociaux. Les entreprises multinationales sont d'autant mieux à même de promouvoir le développement durable que les échanges et les investissements s'effectuent dans le cadre de marchés ouverts, concurrentiels et convenablement réglementés.

De nombreuses entreprises multinationales ont démontré que le respect de normes strictes de conduite pouvait améliorer la croissance. Dans le 
monde d'aujourd'hui, la concurrence est intense et les entreprises multinationales font face à des paramètres différents, qu'ils soient juridiques, sociaux ou réglementaires. Dans ce contexte, des entreprises pourraient être tentées de négliger certains principes ou certaines normes de conduite adéquats afin d'obtenir un avantage concurrentiel indu. De telles pratiques émanant d'un petit nombre d'entreprises pourraient remettre en cause la réputation de la majorité d'entre elles et susciter des préoccupations de la part du public.

Beaucoup d'entreprises ont pris en compte ces préoccupations du public en mettant sur pied des programmes internes et des dispositifs d'orientation et de gestion qui étayent leurs engagements de citoyenneté, de respect de bonnes pratiques et de bonne conduite de l'entreprise et de ses salariés. Certaines ont fait appel à des services de conseil, d'audit et de certification, ce qui a contribué à l'accumulation d'un savoir-faire dans ce domaine. Les entreprises ont également favorisé le dialogue social sur ce qui constitue une conduite responsable des entreprises et elles ont coopéré avec les parties prenantes, dans le cadre d'initiatives pluripartites, afin d'élaborer des orientations relatives à une conduite responsable des entreprises. Les Principes directeurs clarifient les attentes communes aux pouvoirs publics des pays qui y adhèrent et ont en outre valeur de référence pour les entreprises comme pour les autres parties prenantes. Par conséquent, ils complètent et renforcent les initiatives privées qui visent à définir et à faire appliquer des règles de conduite responsable pour les entreprises.

Les gouvernements coopèrent, entre eux et avec d'autres acteurs, pour renforcer le cadre international, juridique et réglementaire, dans lequel les entreprises exercent leurs activités. On peut faire remonter ce processus aux travaux engagés par l'Organisation internationale du travail au début du vingtième siècle. L'adoption par les Nations unies, en 1948, de la Déclaration universelle des droits de l'homme est aussi à marquer d'une pierre blanche. Elle a été suivie par l'élaboration continue de normes applicables dans de nombreux domaines relevant de la responsabilité des entreprises, laquelle se poursuit encore aujourd'hui. L'OCDE a apporté une contribution de poids à ce processus en édictant des normes applicables valables dans des domaines tels que l'environnement, la lutte contre la corruption, les intérêts des consommateurs, le gouvernement d'entreprise ou encore la fiscalité.

L'objectif commun des gouvernements souscrivant aux Principes directeurs est d'encourager la contribution positive que les entreprises multinationales peuvent apporter au progrès économique, environnemental et social, et de réduire au minimum les difficultés que leurs diverses activités peuvent engendrer. Pour atteindre cet objectif, les gouvernements travaillent en partenariat avec les nombreuses entreprises, organisations 
syndicales et autres organisations non gouvernementales qui œuvrent à leur manière aux mêmes fins. Les pouvoirs publics peuvent y contribuer en mettant en place des cadres d'action nationaux efficaces reposant sur plusieurs volets : une politique macro-économique stable, un traitement non discriminatoire des entreprises, une réglementation judicieuse et une surveillance prudentielle adéquate, une justice et une application des lois impartiales ainsi qu'une administration publique efficace et intègre. Ils peuvent aussi y concourir en appliquant et en incitant à adopter des normes et des politiques propices à un développement durable, et en procédant sans relâche à des réformes visant à assurer l'efficience et l'efficacité des activités du secteur public. Les gouvernements souscrivant aux Principes directeurs s'engagent à améliorer continuellement leurs politiques intérieures et internationales dans le but d'accroître le bien-être et de relever le niveau de vie de tous. 


\section{Concepts et principes}

1. Les Principes directeurs sont des recommandations que les gouvernements adressent conjointement aux entreprises multinationales. Ils énoncent des principes et des normes de bonnes pratiques conformes aux législations en vigueur et aux autres normes internationalement admises. Le respect des Principes directeurs par les entreprises est volontaire et n'est pas juridiquement contraignant. Néanmoins, certains sujets abordés dans les Principes directeurs peuvent également être réglementés par des législations nationales ou des engagements internationaux.

2. Les entreprises ont pour obligation première de se conformer à la législation de leur pays. Les Principes directeurs ne sauraient se substituer à une législation ou une réglementation nationale, ni prévaloir sur elles. Si les Principes directeurs vont au-delà de la loi dans de nombreux cas, ils ne devraient pas - et tel n'est pas leur but - placer les entreprises dans une situation où elles feraient face à des obligations contradictoires. Dans les pays où la législation ou la réglementation intérieure contredisent les principes et les normes énoncés dans les Principes directeurs, les entreprises devraient rechercher le moyen de respecter ces principes et ces normes dans toute la mesure du possible sans toutefois risquer de contrevenir à leur législation nationale

3. Dans la mesure où les entreprises multinationales exercent leurs activités dans le monde entier, la coopération internationale dans ce domaine devrait s'étendre à tous les pays. Les gouvernements souscrivant aux Principes directeurs encouragent les entreprises opérant sur leur territoire à respecter les Principes directeurs partout où elles exercent leurs activités, en tenant compte de la situation particulière de chaque pays d'accueil.

4. Une définition précise des entreprises multinationales n'est pas nécessaire pour les besoins des Principes directeurs. Ces entreprises sont en effet présentes dans tous les secteurs de l'économie. Il s'agit généralement d'entreprises ou d'autres entités établies dans plusieurs pays et liées de telle façon qu'elles peuvent coordonner leurs activités de diverses manières. Une ou plusieurs de ces entités peuvent être en 
mesure d'exercer une grande influence sur les activités des autres, mais leur degré d'autonomie au sein de l'entreprise peut être très variable d'une multinationale à l'autre. Leur actionnariat peut être privé, public ou mixte. Les Principes directeurs s'adressent à toutes les entités qui composent l'entreprise multinationale (sociétés mères et/ou entités locales). En fonction de la répartition effective des responsabilités entre elles, on attend des différentes entités qu'elles coopèrent et se prêtent mutuellement concours pour faciliter l'observation des Principes directeurs.

5. Les Principes directeurs ne visent pas à instaurer des différences de traitement entre les entreprises multinationales et les entreprises nationales; ils traduisent des pratiques pouvant être recommandées à toutes. On attend donc des entreprises multinationales et nationales qu'elles aient le même comportement dans tous les cas où les Principes directeurs s'appliquent aux unes et aux autres.

6. Les gouvernements souhaitent encourager un respect aussi large que possible des Principes directeurs. Tout en reconnaissant que les petites et moyennes entreprises peuvent ne pas avoir les mêmes moyens que les grandes, les gouvernements adhérant aux Principes directeurs les encouragent néanmoins à respecter dans toute la mesure du possible les recommandations qu'ils contiennent.

7. Les gouvernements souscrivant aux Principes directeurs ne doivent pas s'en servir à des fins protectionnistes ni d'une manière qui mette en cause l'avantage comparatif d'un pays où les entreprises multinationales investissent.

8. Les gouvernements ont le droit de réglementer les conditions d'activité des entreprises multinationales dans les limites de leurs compétences, sous réserve du respect du droit international. Les entités d'une entreprise multinationale situées dans divers pays sont soumises aux lois en vigueur dans ces pays. Si des entreprises multinationales sont soumises à des obligations contradictoires de la part de pays adhérents ou de pays tiers, les pouvoirs publics concernés sont invités à coopérer en toute bonne foi afin de résoudre les problèmes susceptibles de se poser.

9. Les gouvernements souscrivant aux Principes directeurs les ont établis en prenant l'engagement d'assumer la responsabilité qui leur incombe de traiter les entreprises d'une façon équitable et conformément au droit international et à leurs obligations contractuelles. 
10. Le recours à des mécanismes internationaux adéquats de règlement des différends, y compris l'arbitrage, est encouragé afin de faciliter le règlement des problèmes juridiques susceptibles de surgir entre les entreprises et les autorités des pays d'accueil.

11. Les gouvernements souscrivant aux Principes directeurs conviennent de les mettre en œuvre et d'en encourager l'usage. Ils créeront des Points de contact nationaux qui auront pour tâche de promouvoir les Principes directeurs et serviront de forum de discussion pour toutes les questions concernant les Principes directeurs. En outre, les gouvernements adhérents participeront à des procédures adéquates d'examen et de consultation pour les questions concernant l'interprétation des Principes directeurs dans un monde en mutation. 


\section{Principes généraux}

Les entreprises doivent tenir pleinement compte des politiques établies dans les pays où elles exercent leurs activités et prendre en considération les points de vue des autres acteurs. A cet égard :

A. Les entreprises devraient :

1. Contribuer aux progrès économiques, environnementaux et sociaux en vue de parvenir à un développement durable.

2. Respecter les droits de l'homme internationalement reconnus vis-à-vis des personnes affectées par leurs activités.

3. Encourager le renforcement de capacités au niveau local en coopérant étroitement avec la communauté locale, y compris les milieux d'affaires, tout en développant les activités de l'entreprise sur le marché intérieur et sur les marchés extérieurs d'une manière compatible avec de saines pratiques commerciales.

4. Encourager la formation de capital humain, en particulier en créant des possibilités d'emploi et en facilitant la formation des salariés.

5. S'abstenir de rechercher ou d'accepter des exceptions non prévues dans le dispositif législatif ou réglementaire concernant les droits de l'homme, l'environnement, la santé, la sécurité, le travail, la fiscalité, les incitations financières ou d'autres domaines.

6. Appuyer et faire observer des principes de bon gouvernement d'entreprise et mettre au point et appliquer de bonnes pratiques de gouvernement d'entreprise, y compris au sein des groupes d'entreprises.

7. Élaborer et appliquer des pratiques d'autodiscipline et des systèmes de gestion efficaces qui favorisent une relation de confiance mutuelle entre les entreprises et les sociétés dans lesquelles elles exercent leurs activités.

8. Faire en sorte que les travailleurs qu'elles emploient soient bien au fait des politiques qu'elles ont mises en place et les inciter à s'y conformer 
en les diffusant comme il convient, notamment par des programmes de formation.

9. S'abstenir d'engager des actions discriminatoires ou disciplinaires à l'encontre des travailleurs qui auraient, de bonne foi, rapporté à la direction ou, le cas échéant, aux autorités publiques compétentes, des informations sur des pratiques contraires à la loi, aux Principes directeurs ou aux politiques de l'entreprise.

10. Exercer une diligence raisonnable fondée sur les risques, par exemple en intégrant cette dimension dans leurs systèmes de gestion des risques, afin d'identifier, de prévenir ou d'atténuer les incidences négatives, réelles ou potentielles, décrites dans les paragraphes 11 et 12, et rendre compte de la manière dont elles répondent à de telles incidences. La nature et la portée de la diligence raisonnable dépendent des circonstances propres à une situation particulière.

11. Éviter d'avoir, du fait de leurs propres activités, des incidences négatives dans des domaines visés par les Principes directeurs, ou d'y contribuer, et prendre des mesures qu'imposent ces incidences lorsqu'elles se produisent.

12. S'efforcer d'empêcher ou d'atténuer une incidence négative, dans le cas où elles n'y ont pas contribué mais où cette incidence est néanmoins directement liée à leurs activités, à leurs produits ou à leurs services en vertu d'une relation d'affaires. Ceci ne doit pas être interprété comme transférant la responsabilité de l'entité à l'origine d'une incidence négative sur l'entreprise avec laquelle elle entretient une relation d'affaires.

13. En plus de répondre à des incidences négatives dans des domaines visés par les Principes directeurs, encourager dans la mesure du possible leurs partenaires commerciaux, y compris leurs fournisseurs et leurs soustraitants, à appliquer des principes de conduite responsable conformes aux Principes directeurs.

14. S'engager auprès des parties prenantes concernées en leur donnant de réelles possibilités de faire valoir leurs points de vue lorsqu'il s'agit de planifier et de prendre des décisions relatives à des projets ou d'autres activités susceptibles d'avoir un impact significatif sur les populations locales.

15. S'abstenir de toute ingérence indue dans les activités politiques locales. 


\section{B. Les entreprises sont encouragées à :}

1. Soutenir, en fonction des circonstances qui leur sont propres, les efforts concertés déployés dans les enceintes appropriées pour favoriser la liberté sur l'Internet, notamment par le respect de la liberté d'expression, de réunion et d'association en ligne.

2. Participer ou apporter leur soutien, le cas échéant, à des initiatives privées ou multipartites et au dialogue social sur une gestion responsable de la chaîne d'approvisionnement, en s'assurant que ces initiatives prennent dûment en compte leurs conséquences sociales ou économiques sur les pays en développement et respectent les normes internationalement admises existantes

\section{Commentaire sur les principes généraux}

1. Le chapitre des Principes directeurs consacré aux principes généraux est le premier qui contienne des recommandations précises adressées aux entreprises. Il est important en ce sens qu'il fixe l'orientation générale et établit des principes fondamentaux communs pour les recommandations spécifiques des chapitres suivants.

2. Les entreprises sont encouragées à coopérer avec les pouvoirs publics pour la mise au point et l'application des politiques et des réglementations. Prendre en considération les points de vue des autres acteurs de la société peut également enrichir ce processus ; on vise ici la communauté locale et les milieux d'affaires. Il est également admis que les pouvoirs publics doivent faire preuve de transparence dans leurs rapports avec les entreprises et les consulter sur ces mêmes questions. Les entreprises doivent être considérées comme des partenaires des pouvoirs publics pour la mise au point et l'application des dispositifs volontaires et réglementaires (dont les Principes directeurs constituent un élément) ayant trait aux politiques qui les concernent.

3. Il ne saurait y avoir de contradiction entre les activités des entreprises multinationales et le développement durable. C'est précisément cette complémentarité que les Principes directeurs entendent favoriser. De fait, il est fondamental que des liens existent entre les progrès économiques, sociaux et environnementaux pour conforter l'objectif du développement durable ${ }^{4}$.

4. L'une des définitions les plus largement acceptées du développement durable a été donnée en 1987 par la Commission mondiale sur l'environnement et le développement (Commission «Brundtland»). Il s'agit d'un «développement qui 
4. Le chapitre 4 reprend de manière plus détaillée les recommandations générales relatives aux droits de l'homme formulées au paragraphe A.2.

5. Par ailleurs, les Principes directeurs reconnaissent et encouragent la contribution que les entreprises multinationales peuvent apporter à la création de capacités locales en raison de leurs activités au niveau local. De même, la recommandation concernant la formation de capital humain est résolument tournée vers l'avenir, en reconnaissant expressément la contribution que les entreprises multinationales peuvent offrir à leurs travailleurs sur le plan du développement humain individuel. Cette recommandation n'a pas seulement trait à l'embauche ; elle englobe également la formation et les autres actions de développement professionnel. La formation de capital humain couvre également la notion de non-discrimination au niveau de l'embauche, de la promotion, de l'apprentissage tout au long de la vie et des autres formules de formation en cours d'emploi.

6. Les Principes directeurs préconisent que, d'une manière générale, les entreprises évitent de chercher à obtenir des exceptions non prévues dans le dispositif législatif ou réglementaire ayant trait aux droits de l'homme, à l'environnement, à la santé, à la sécurité, au travail, à la fiscalité ou aux incitations financières, pour n'en citer que quelques-uns, mais sans préjudice du droit qu'elles ont par ailleurs de chercher à faire évoluer un cadre législatif ou réglementaire. L'expression «ou d'accepter » souligne par ailleurs le rôle de l'État qui peut avoir proposé les exceptions. Une disposition de ce type s'adresse traditionnellement aux pouvoirs publics, mais elle concerne également les entreprises multinationales directement. Il faut néanmoins garder à l'esprit que, dans certains cas, des exceptions particulières à des lois ou à d'autres mesures peuvent être conformes à ces lois pour des raisons légitimes d'intérêt public. On en trouvera des exemples dans les chapitres consacrés à l'environnement ou à la politique de la concurrence.

7. Aux termes des Principes directeurs, il est recommandé que les entreprises mettent en œuvre de saines pratiques de gouvernement d'entreprise inspirées des Principes de gouvernement d'entreprise de $l^{\prime} O C D E$. Elles sont en particulier appelées à protéger et à faciliter l'exercice des droits des actionnaires, notamment le droit à un traitement équitable. Les entreprises devraient reconnaître les droits des parties prenantes tels que définis dans des textes législatifs ou des accords mutuels et encourager une coopération active avec ces parties

répond aux besoins du présent sans compromettre la capacité des générations futures de répondre aux leurs ». 
prenantes pour favoriser la création de richesses et d'emplois et assurer la viabilité d'entreprises financièrement saines.

8. Selon les Principes directeurs, le conseil d'administration d'une entitémère doit assurer le pilotage stratégique de l'entreprise, et en contrôler efficacement la direction. Le conseil d'administration doit aussi rendre des comptes à l'entreprise et aux actionnaires, tout en veillant aux intérêts des parties prenantes. Lorsqu'il s'acquitte de ces responsabilités, le conseil d'administration doit s'assurer de l'intégrité des systèmes de comptabilité et de communication financière de l'entreprise, et notamment de l'indépendance de la vérification des comptes, de l'existence de dispositifs de contrôle adéquats, en particulier pour la gestion des risques et le contrôle financier et opérationnel, ainsi que du respect du droit et des normes applicables.

9. Les Principes directeurs visent aussi les groupes d'entreprises, même si le conseil d'administration des filiales peut être tenu par des obligations découlant de la législation du pays où elles sont constituées en société. Les dispositifs de conformité et de contrôle doivent aussi intéresser ces filiales dans la mesure du possible. De plus, la mission de surveillance des pratiques de gouvernance qui incombe au conseil implique également de contrôler en permanence les structures internes d'un groupe afin de s'assurer que les responsabilités y sont clairement définies partout.

10. Les entreprises multinationales publiques sont soumises aux mêmes recommandations que les entreprises privées, mais la surveillance exercée par l'État est souvent démultipliée lorsque celui-ci est le propriétaire final. Les Lignes directrices de l'OCDE sur le gouvernement d'entreprise des entreprises publiques constituent pour ces sociétés un guide utile et personnalisé et les recommandations qui y sont énoncées sont de nature à améliorer sensiblement leur gouvernance.

11. Bien que les pouvoirs publics soient au premier chef responsables de l'amélioration du cadre juridique et institutionnel de la réglementation, les entreprises ont aussi d'excellentes raisons commerciales de mettre en place un gouvernement d'entreprise de qualité.

12. Il existe un arsenal de plus en plus étoffé d'instruments et de mesures émanant des organisations professionnelles qui abordent bien des aspects de la conduite des entreprises et de leurs relations avec la collectivité. A cet égard, des évolutions intéressantes sont en cours dans le secteur financier. Les entreprises reconnaissent que leurs activités ont souvent une incidence sur la société et sur l'environnement. La mise en place de pratiques d'autodiscipline et de systèmes de gestion par les 
entreprises soucieuses de réaliser les objectifs en question - en contribuant ainsi au développement durable - en offre un exemple. Développer ces pratiques ne peut que contribuer à des relations fructueuses entre les entreprises et les sociétés dans lesquelles elles opèrent.

13. Puisqu'on attend des entreprises des pratiques efficaces d'autodiscipline, on attend également d'elles une sensibilisation de leurs salariés aux mesures qu'elles mettent en œuvre. Des mécanismes de protection des salariés qui «donnent l'alerte» de bonne foi sont également recommandés, y compris pour protéger les salariés qui, en l'absence de mesures réparatrices promptes ou confrontés à un risque raisonnable de conséquences négatives pour leur emploi, signalent aux autorités publiques compétentes des pratiques contraires à la loi. Bien qu'une telle protection concerne tout particulièrement les mesures de lutte contre la corruption et de protection de l'environnement, elle s'applique également aux autres recommandations des Principes directeurs.

14. Dans les Principes directeurs, on entend par diligence raisonnable le processus qui, en tant que partie intégrante de leurs systèmes de prise de décisions et de gestion des risques, permet aux entreprises d'identifier, de prévenir et d'atténuer les incidences négatives, réelles ou potentielles, de leurs activités, ainsi que de rendre compte de la manière dont elles abordent cette question. La diligence raisonnable peut être intégrée dans d'autres systèmes de gestion de risque au sein de l'entreprise à condition qu'elle aille au-delà de l'identification et la gestion des risques significatifs pour l'entreprise elle-même, pour englober également les risques d'incidences négatives dans des domaines visés par les Principes directeurs. Les incidences potentielles doivent être traitées grâce à des mesures de prévention ou d'atténuation, tandis que les incidences réelles doivent être traitées grâce à des mesures de réparation. Les Principes directeurs concernent les incidences négatives dont les entreprises sont à l'origine ou auxquelles elles contribuent, ou qui sont directement liées à leurs activités, leurs produits et leurs services du fait de l'existence d'une relation d'affaires, telle qu'elle est définie aux paragraphes A.11 et A.12. En exerçant une diligence raisonnable, les entreprises peuvent mieux se prémunir contre le risque de provoquer de telles incidences négatives. Aux fins de la présente recommandation, «contribuer à » une incidence négative doit être interprété comme une contribution substantielle, c'est-à-dire une activité qui provoque, facilite ou incite une autre entité à provoquer une incidence négative, et n'inclut pas les contributions mineures ou négligeables. On entend par «relation d'affaires » toute relation avec des partenaires commerciaux, des entités appartenant à la châne 
d'approvisionnement ou toute autre entité, publique ou non, directement liée à ses activités, ses produits ou ses services. La recommandation exprimée au paragraphe A.10 s'applique aux questions ayant trait aux incidences négatives couvertes par les Principes directeurs. Elle ne s'applique pas aux chapitres consacrés à la science et à la technologie, à la concurrence et à la fiscalité.

15. La nature et la portée de la diligence raisonnable (telles que les mesures spécifiques à prendre) appropriée à une situation particulière dépendront de facteurs tels que la taille de l'entreprise, le contexte dans lequel s'inscrivent ses activités, les recommandations spécifiques des Principes directeurs et la gravité des incidences négatives. Des recommandations portant spécifiquement sur la diligence raisonnable en matière de droits de l'homme figurent au chapitre IV.

16. Lorsque les entreprises ont un grand nombre de fournisseurs, elles sont invitées à identifier les domaines généraux dans lesquels le risque d'incidences négatives est le plus significatif puis, à partir de cette évaluation du risque, à exercer la diligence raisonnable de manière prioritaire à l'égard de certains fournisseurs.

17. Le fait, pour les entreprises, d'éviter d'avoir, du fait de leurs propres activités, des incidences négatives dans des domaines visés par les Principes directeurs, ou d'y contribuer, inclut leurs activités dans la chaîne d'approvisionnement. Les relations dans la chaîne d'approvisionnement peuvent prendre diverses formes, par exemple des franchises, des accords de licence ou de sous-traitance. Les entités dans la chaîne d'approvisionnement sont souvent elles-mêmes des entreprises multinationales et, de ce fait, celles qui exercent leurs activités dans ou à partir de pays adhérant à la Déclaration sont couvertes par les présents Principes directeurs.

18. Dans le contexte de la chaîne d'approvisionnement, si une entreprise s'aperçoit qu'il existe un risque d'incidence négative, elle devrait alors prendre les mesures nécessaires pour y mettre fin ou pour l'empêcher.

19. Si une entreprise s'aperçoit qu'elle risque de contribuer à une incidence négative, elle devrait alors prendre les mesures nécessaires pour interrompre ou pour empêcher cette contribution et user de son influence pour atténuer les incidences résiduelles dans toute la mesure du possible. On considère qu'il y a influence lorsqu'une entreprise a la capacité de faire modifier les pratiques néfastes de l'entité responsable du dommage.

20. Bien répondre à l'attente exprimée au paragraphe A.12 signifie qu'une entreprise, seule ou en collaboration avec d'autres entités, le cas 
échéant, devrait utiliser son influence pour intervenir auprès de l'entité responsable de l'incidence négative afin de prévenir ou d'atténuer cette incidence.

21. Les Principes directeurs reconnaissent qu'il existe des limites pratiques concernant la capacité des entreprises à amener leurs fournisseurs à changer de comportement. Cette limitation tient aux caractéristiques des produits, au nombre des fournisseurs, à la structure et à la complexité de la chaîne d'approvisionnement, ou encore à la position de marché de l'entreprise vis-à-vis de ses fournisseurs ou d'autres entités de la chaîne d'approvisionnement. Cela étant, les entreprises peuvent aussi influencer leurs fournisseurs par le biais d'accords contractuels tels que des contrats de gestion, des obligations de pré-qualification pour les fournisseurs potentiels, des conventions de vote ou encore des accords de licence ou de franchise. Parmi d'autres facteurs pouvant être pris en compte pour déterminer la réponse adéquate à des risques identifiés, on peut citer la gravité des incidences négatives et la probabilité qu'elles surviennent, ou encore le degré d'importance du fournisseur pour l'entreprise.

22. Du point de vue de la relation d'affaires, les réponses appropriées peuvent prendre plusieurs formes: poursuite de la relation avec le fournisseur pendant toute la durée des efforts d'atténuation des risques ; suspension temporaire de la relation avec poursuite des efforts d'atténuation des risques ; ou, en dernier recours, rupture de la relation avec le fournisseur, soit après que les efforts d'atténuation des risques ont échoué, soit parce que l'entreprise estime qu'aucune atténuation n'est possible, soit à cause de la gravité de l'incidence négative. L'entreprise devrait aussi prendre en compte les incidences négatives éventuelles, sur les plans social et économique, d'une décision de désengagement.

23. Les entreprises peuvent aussi s'engager aux côtés des fournisseurs et d'autres entités de la chaîne d'approvisionnement pour améliorer leurs performances, en coopération avec d'autres parties prenantes, notamment grâce à la formation du personnel ou à d'autres formes de renforcement des capacités, et pour les aider à intégrer, dans leurs pratiques professionnelles, des principes de conduite responsable compatibles avec les Principes directeurs. Lorsque des fournisseurs ont de nombreux clients et sont potentiellement confrontés à des obligations contradictoires imposées par des acheteurs différents, les entreprises sont encouragées, en tenant dûment compte des préoccupations relatives à la concurrence, à participer aux efforts collectifs déployés à l'échelle de leur secteur d'activité aux côtés des autres entreprises avec lesquelles elles ont des fournisseurs communs afin de coordonner leurs politiques 
vis-à-vis de la chaîne d'approvisionnement et leurs stratégies de gestion des risques, y compris grâce à des échanges d'informations.

24. Les entreprises sont également encouragées à participer à des initiatives privées ou multipartites et au dialogue social sur une gestion responsable de la chaîne d'approvisionnement, telles que celles qui sont engagées dans le cadre de «l'agenda proactif » du Comité préconisé par la Décision du Conseil de l'OCDE sur les Principes directeurs de l'OCDE à l'intention des entreprises multinationales et aux Lignes de procédure figurant en annexe.

25. L'engagement des parties prenantes repose sur des procédures interactives avec les parties prenantes concernées grâce par exemple à des réunions, des auditions ou des consultations. Un engagement efficace des parties prenantes suppose une communication à double sens et la bonne foi de tous les participants. Un tel engagement peut être particulièrement utile lorsqu'il s'agit de planifier et de prendre des décisions sur des projets ou d'autres activités impliquant par exemple une utilisation intensive de terres ou d'eau, susceptibles d'avoir des retombées significatives sur les populations locales.

26. Le paragraphe B.1 prend acte de l'émergence d'une question importante. Il ne vise pas à instaurer de nouvelles normes, mais ne préjuge pas non plus de l'élaboration de normes nouvelles. Il prend acte du fait que les entreprises ont des intérêts qui seront affectés et qu'en participant, aux côtés d'autres parties prenantes, à l'examen des questions soulevées, elles-mêmes et d'autres pourront mieux comprendre ces questions et apporter ainsi une contribution positive. Il admet que ces questions comportent sans doute plusieurs aspects et souligne que la coopération doit être recherchée au sein des enceintes appropriées. Il ne préjuge pas des positions adoptées par les différents pays dans le domaine du commerce électronique au sein de l'Organisation mondiale du commerce (OMC). Il n'a pas pour objet de négliger d'autres intérêts importants de l'action publique liés à l'utilisation de l'Internet qui devraient être pris en compte. ${ }^{5}$ Enfin, comme c'est le cas plus généralement pour les Principes directeurs, il n'a pas pour objet d'imposer aux entreprises des obligations contradictoires, conformément aux paragraphes 2 et 8 du chapitre «Concepts et principes » des Principes directeurs.

27. Enfin, il faut noter que les mesures d'autodiscipline et les mesures du même type, y compris les Principes directeurs, ne doivent pas de l'information. 
restreindre illégalement la concurrence et ne doivent pas non plus être considérées comme se substituant à une législation ou une réglementation publique efficaces. Il est entendu que les entreprises multinationales doivent éviter tout effet de distorsion des échanges et des investissements qui pourrait résulter des codes ou des pratiques d'autodiscipline qu'elles mettent au point. 


\section{Publication d'informations}

1. Les entreprises devraient s'assurer de la publication, dans les délais requis, d'informations exactes sur tous les aspects significatifs de leurs activités, de leur structure, de leur situation financière, de leurs résultats, de leur actionnariat et de leur système de gouvernement d'entreprise. Ces informations devraient être fournies pour l'entreprise dans son ensemble et, s'il y a lieu, par branche d'activité ou zone géographique. Les politiques de publication d'informations des entreprises devraient être adaptées à leur nature, à leur taille et au lieu de leur implantation, en tenant compte du coût, de la confidentialité et d'autres considérations relevant de la concurrence.

2. Dans leurs politiques de publication d'informations, les entreprises devraient (sans que la liste suivante soit limitative) prévoir de publier des informations détaillées concernant :

a) leurs résultats financiers et leurs résultats d'exploitation ;

b) leurs objectifs ;

c) les participations significatives et le détail des droits de vote, y compris la structure des groupes d'entreprise et les relations intragroupe, ainsi que les mécanismes de renforcement du contrôle ;

d) la politique de rémunération des membres du conseil d'administration et des principaux dirigeants, avec des informations sur les administrateurs, en particulier leurs qualifications, le processus mis en œuvre pour leur nomination, leur appartenance éventuelle au conseil d'administration d'autres sociétés et l'appréciation du conseil d'administration sur leur indépendance ;

e) les transactions avec des parties liées ;

f) les facteurs de risque prévisibles ;

g) les questions relatives aux travailleurs et aux autres parties prenantes ;

h) les structures et les politiques de gouvernement d'entreprise, en particulier le contenu de tout code ou stratégie de gouvernement 
d'entreprise élaboré par la société ainsi que la procédure destinée à en assurer la mise en œuvre.

3. Les entreprises sont encouragées à communiquer des informations supplémentaires pouvant inclure :

a) des déclarations de principes ou des règles de conduite à l'intention du public, y compris, si leurs activités le justifient, des informations relatives à leurs politiques vis-à-vis des thèmes abordés dans les Principes directeurs ;

b) des politiques ou autres codes de conduite auxquels elles souscrivent, avec la date de leur adoption et la mention des pays et des entités auxquels ils s'appliquent ;

c) leurs performances en matière de respect de ces déclarations ou codes ;

d) des informations sur les dispositifs d'audit interne, de gestion des risques et d'application de la loi ;

e) des informations sur les relations avec les travailleurs et les autres parties prenantes.

4. Les entreprises devraient respecter des normes de haute qualité en matière de publication d'informations comptables, financières et non financières, y compris d'informations environnementales et sociales le cas échéant. Les normes ou les politiques de recueil et de publication des informations devraient être communiquées. Les comptes devraient être vérifiés chaque année par un réviseur comptable indépendant, compétent et qualifié, chargé de donner au conseil d'administration et aux actionnaires un avis extérieur objectif certifiant que les états financiers donnent une image fidèle de la situation financière et des résultats de la société sous tous leurs aspects significatifs.

\section{Commentaire sur la publication d'informations}

28. Ce chapitre a pour objet de favoriser une meilleure compréhension des activités des entreprises multinationales. Des informations claires et complètes sur les entreprises sont importantes pour une multitude d'utilisateurs, depuis les actionnaires et la communauté financière jusqu'aux travailleurs, en passant par les communautés locales, les groupes d'intérêts, les pouvoirs publics ou la société dans son ensemble. Pour que l'on puisse mieux connaître les entreprises et leurs interactions avec la société et l'environnement, il faut qu'elles exercent leurs 
activités dans la transparence et répondent aux demandes d'information d'un public de plus en plus exigeant.

29. Les informations citées dans le présent chapitre répondent de deux manières à l'obligation de publication d'informations. La première série de recommandations reprend les éléments qui sont mentionnés dans les Principes de gouvernement d'entreprise de l'OCDE. Les notes les concernant contiennent des orientations complémentaires et les recommandations énoncées dans les Principes directeurs devraient en être rapprochés. Le premier ensemble de recommandations en matière de publication d'informations pourrait être complété par un second ensemble de recommandations sur le même thème que les entreprises seront invitées à suivre. Ces recommandations visent en priorité les entreprises cotées, mais si elles sont jugées applicables compte tenu de la nature, de la taille et de l'emplacement des entreprises concernées, elles pourraient en outre contribuer fort utilement à améliorer le gouvernement d'entreprise des sociétés non cotées, privées ou publiques.

30. Les recommandations en matière de publication d'informations ne sont pas censées imposer aux entreprises une charge excessive en termes de coûts ou de lourdeurs administratives. On n'attend pas non plus des entreprises qu'elles rendent publiques des informations pouvant compromettre leur situation concurrentielle, à moins que ces informations ne soient indispensables pour éclairer pleinement les investisseurs et éviter de les induire en erreur. Pour définir quelles sont les informations qui devraient au minimum être publiées, les Principes directeurs utilisent la notion de caractère significatif. On considère qu'une information est significative si le fait de ne pas la publier ou de la fausser risquerait d'influencer les décisions économiques des personnes auxquelles elle est destinée.

31. De plus, selon les Principes directeurs, il convient généralement d'établir et de publier ces informations en respectant des normes de grande qualité en matière de comptabilité et de communication financière et non financière. Cela permet d'améliorer de manière significative la capacité des investisseurs à suivre l'entreprise, parce que les informations publiées gagnent en fiabilité et en comparabilité et parce que les performances de l'entreprise sont plus faciles à saisir. La vérification annuelle des comptes par un réviseur comptable indépendant préconisée dans les Principes directeurs devrait également contribuer à un meilleur contrôle et à un plus grand degré de conformité de la part des entreprises. 
32. La publication d'informations est abordée sous deux angles. Le premier ensemble de recommandations appelle les entreprises à publier, dans les délais requis, des informations exactes sur tous leurs aspects significatifs, en particulier leur situation financière, leurs résultats, leur actionnariat et leur système de gouvernement d'entreprise. On attend également des entreprises qu'elles publient des informations suffisantes concernant la rémunération des membres des organes d'administration et des principaux dirigeants (de manière individuelle ou globale), afin que les investisseurs puissent évaluer correctement les coûts et avantages des plans de rémunération et le rôle que jouent dans les résultats de l'entreprise les dispositifs incitatifs comme l'attribution d'options d'achat d'actions. Les transactions avec des parties liées et les facteurs de risque significatifs prévisibles doivent également être divulgués, de même que les questions importantes concernant les travailleurs et les autres parties prenantes.

33. Les Principes directeurs encouragent également une deuxième catégorie de pratiques de publication ou de communication portant sur des domaines où les normes d'information sont encore en train d'évoluer (par exemple données sociales, informations environnementales ou informations concernant les risques). C'est le cas, en particulier, pour les émissions de gaz à effet de serre, dans la mesure où le champ d'application de leur suivi s'étend aux émissions directes et indirectes, actuelles et futures, au niveau de chaque entreprise et de chaque produit; la biodiversité constitue un autre exemple. De nombreuses entreprises fournissent des informations qui vont au-delà des seuls résultats financiers et considèrent que la publication de ces informations est un bon moyen de manifester leur attachement à des pratiques socialement acceptables. Dans certains cas, ce deuxième type de publication d'informations - ou de communication avec le public et les autres parties directement affectées par les activités de l'entreprise - peut s'appliquer à des entités allant au-delà de celles couvertes par la comptabilité financière de l'entreprise. Il peut également s'étendre, par exemple, à des informations sur les activités de sous-traitants et de fournisseurs, ou de partenaires dans le cadre de co-entreprises. Ceci est particulièrement approprié pour contrôler le transfert, à des partenaires, d'activités dommageables pour l'environnement.

34. Beaucoup d'entreprises ont adopté des mesures qui ont pour but de les aider à se conformer à la loi et à certaines normes de conduite, et aussi à améliorer la transparence de leurs activités. De plus en plus nombreuses sont celles qui ont mis en place des codes de conduite volontaires, traduisant leur adhésion à certaines valeurs éthiques dans des domaines comme l'environnement, les droits de l'homme, les normes du travail, 
la protection des consommateurs ou la fiscalité. Des systèmes de gestion spécialisés ont été élaborés ou sont en cours de mise au point et continuent à évoluer pour les aider à respecter ces engagements; ils portent en particulier sur des systèmes d'informations, des procédures opérationnelles ou encore des obligations en matière de formation. Les entreprises coopèrent avec les ONG et les organisations intergouvernementales à la mise au point de normes de publication d'informations grâce auxquelles elles seront mieux à même de rendre compte de l'influence de leurs activités dans les domaines qui se rapportent au développement durable [dans le cadre, par exemple, de l'Initiative mondiale sur les rapports de performance («Global Reporting Initiative »)].

35. On encourage les entreprises à rendre l'accès aux informations publiées facile et peu coûteux et à envisager d'utiliser à cet effet les technologies de l'information. Les informations communiquées aux usagers sur le marché du pays d'origine devraient être également accessibles à tous les usagers intéressés. Les entreprises peuvent prendre des mesures spéciales pour diffuser ces informations au sein des communautés n'ayant pas accès aux médias imprimés (par exemple les populations pauvres directement affectées par les activités de l'entreprise). 


\section{Droits de l'homme}

Les États ont le devoir de protéger les droits de l'homme. Dans le cadre des droits de l'homme internationalement reconnus, des engagements internationaux envers les droits de l'homme souscrits par les pays où elles exercent leurs activités ainsi que des lois et règlements nationaux pertinents, les entreprises devraient :

1. Respecter les droits de l'homme, ce qui signifie qu'elles doivent se garder de porter atteinte aux droits d'autrui et parer aux incidences négatives sur les droits de l'homme dans lesquelles elles ont une part.

2. Dans le cadre de leurs activités, éviter d'être la cause d'incidences négatives sur les droits de l'homme ou d'y contribuer, et parer à ces incidences lorsqu'elles surviennent.

3. S'efforcer de prévenir et d'atténuer les incidences négatives sur les droits de l'homme directement liées à leurs activités, leurs biens ou leurs services en raison d'une relation d'affaires avec une autre entité, même si elles ne contribuent pas à ces incidences.

4. Élaborer une politique formulant leur engagement à respecter les droits de l'homme.

5. Exercer une diligence raisonnable en matière de droits de l'homme, en fonction de leur taille, de la nature et du contexte de leurs activités et de la gravité des risques d'incidences négatives sur ces droits.

6. Établir des mécanismes légitimes ou s'y associer afin de remédier aux incidences négatives sur les droits de l'homme lorsqu'il s'avère qu'elles en sont la cause ou qu'elles y ont contribué.

\section{Commentaire sur les droits de l'homme}

36. Ce chapitre s'ouvre sur un chapeau qui définit le cadre dans lequel s'inscrivent les recommandations spécifiques relatives au respect des droits de l'homme par les entreprises. Il s'inspire du cadre pour les entreprises et les droits de l'homme "Protéger, respecter et réparer» 
établi par les Nations Unies et est conforme à ses lignes directrices de mise en œuvre.

37. Le chapeau et le premier paragraphe disposent que les États ont le devoir de protéger les droits de l'homme, et que les entreprises, quels que soient leurs taille, secteur d'activité, contexte opérationnel, structure de propriété et organisation, doivent respecter les droits de l'homme partout où elles exercent leurs activités. Le respect des droits de l'homme est la norme mondiale de conduite attendue des entreprises, indépendamment de la capacité et/ou de la volonté des États de satisfaire à leurs obligations en la matière, et ne saurait atténuer ces obligations.

38. Le fait qu'un État n'applique pas sa législation nationale pertinente ou ne respecte pas ses obligations internationales en matière de droits de l'homme, ou qu'il puisse enfreindre cette législation ou ces obligations internationales est sans effet sur la responsabilité des entreprises de respecter les droits de l'homme. Dans les pays où les lois et règlements nationaux sont contraires aux droits de l'homme reconnus internationalement, les entreprises doivent rechercher les moyens de les respecter le plus possible sans violer la législation nationale, conformément au paragraphe 2 du chapitre consacré aux concepts et principes.

39. Dans tous les cas et indépendamment du pays ou du contexte spécifique dans lequel s'inscrivent les activités des entreprises, il faut se référer pour le moins aux droits de l'homme reconnus internationalement exprimés dans la Charte internationale des droits de l'homme, qui comprend la Déclaration universelle des droits de l'homme et les principaux instruments d'après lesquels elle a été codifiée: le Pacte international relatif aux droits civils et politiques et le Pacte international relatif aux droits économiques, sociaux et culturels, ainsi qu'aux principes concernant les droits fondamentaux exposés dans la Déclaration de l'Organisation internationale du travail de 1998 sur les principes et droits fondamentaux au travail.

40. Les entreprises peuvent avoir une incidence sur pratiquement tous les droits de l'homme reconnus internationalement. Dans la pratique, certains droits de l'homme peuvent être plus vulnérables que d'autres dans certains secteurs ou contextes, et feront donc l'objet d'une attention plus soutenue. Toutefois, les situations peuvent changer; par conséquent, tous les droits doivent être périodiquement examinés. En fonction des circonstances, les entreprises commerciales peuvent être amenées à envisager l'adoption de normes supplémentaires. Par exemple, les entreprises doivent respecter les droits de l'homme des 
personnes qui appartiennent à des catégories spécifiques ou de populations qui méritent une attention particulière, dès lors qu'elles risquent d'avoir une incidence négative sur ces droits. Dans ce contexte, les instruments des Nations Unies ont précisé les droits des populations autochtones; des personnes appartenant à des minorités nationales ou ethniques, religieuses et linguistiques; des femmes ; des enfants; des personnes handicapées; et des travailleurs migrants et leurs familles. En outre, dans les situations de conflit armé, les entreprises devraient respecter les normes du droit international humanitaire, ce qui peut les aider à éviter d'avoir des incidences négatives ou d'y contribuer lorsqu'elles exercent des activités dans un environnement aussi difficile.

41. Dans le paragraphe 1, parer aux incidences négatives, réelles et potentielles, sur les droits de l'homme consiste à prendre des mesures adéquates pour repérer, si possible prévenir, et atténuer les incidences potentielles sur les droits de l'homme, parer aux incidences réelles, et rendre compte des mesures prises à cette fin. L'expression «porter atteinte » désigne les incidences négatives qu'une entreprise peut avoir sur les droits de l'homme.

42. Le paragraphe 2 recommande aux entreprises d'éviter d'être la cause d'incidences négatives sur les droits de l'homme ou d'y contribuer par leurs activités et de parer à ces incidences négatives lorsqu'elles surviennent. Le terme «activités » recouvre à la fois les actions et les omissions. Lorsqu'une entreprise a ou est susceptible d'avoir une incidence négative sur les droits de l'homme, elle devrait prendre les mesures nécessaires pour empêcher cette incidence ou y mettre un terme. Lorsqu'une entreprise contribue ou est susceptible de contribuer à une telle incidence, elle devrait prendre les mesures nécessaires pour empêcher cette contribution ou y mettre un terme, ou user de son pouvoir pour atténuer le plus possible l'incidence résiduelle éventuelle. L'entreprise détient un tel pouvoir lorsqu'elle est en mesure de modifier les pratiques d'une entité qui ont des incidences négatives sur les droits de l'homme.

43. Le paragraphe 3 concerne des situations plus complexes dans lesquelles une entreprise n'a pas contribué à une incidence négative sur les droits de l'homme, mais où cette incidence est néanmoins directement liée à ses activités, biens ou services du fait de sa relation d'affaires avec une autre entité. Le paragraphe 3 n'a pas pour but de transférer la responsabilité de l'entité qui est la cause d'une incidence négative sur les droits de l'homme à l'entreprise avec qui elle a une relation d'affaires. Pour répondre à l'intention exprimée dans ce paragraphe, une entreprise, agissant seule ou en coopération avec d'autres entités, le cas échéant, devrait exercer son pouvoir pour convaincre l'entité qui a une 
incidence négative sur les droits de l'homme d'empêcher ou d'atténuer cette incidence. Les « relations d'affaires» d'une entreprise englobent les relations avec ses partenaires commerciaux, les agents de sa chaîne d'approvisionnement, d'autres acteurs non publics et des agents de l'État directement liés à ses activités commerciales, biens ou services. Parmi les facteurs qui entreront en ligne de compte pour déterminer l'action appropriée à engager en pareilles situations figurent le pouvoir exercé par l'entreprise sur l'entité concernée, l'importance de cette relation pour l'entreprise, la gravité de l'incidence, et le point de savoir si la rupture de la relation avec l'entité aurait en soi une incidence négative sur les droits de l'homme.

44. Le paragraphe 4 recommande aux entreprises d'exprimer leur engagement à respecter les droits de l'homme par une déclaration qui : (i) soit approuvée au niveau hiérarchique le plus élevé de l'entreprise ; (ii) soit établie par des spécialistes internes et/ou externes; (iii) définisse les attentes en matière de respect des droits de l'homme de la part du personnel, des partenaires commerciaux et d'autres parties directement associées aux activités, biens ou services de l'entreprise ; (iv) soit diffusée publiquement et communiquée en interne et en externe à l'ensemble du personnel, des partenaires commerciaux et des autres parties concernées; (v) se traduise par des politiques et procédures opérationnelles qui donnent à cet engagement une dimension concrète.

45. Le paragraphe 5 recommande aux entreprises d'exercer une diligence raisonnable en matière de droits de l'homme. Ce processus implique d'évaluer les incidences réelles et potentielles sur les droits de l'homme, d'intégrer les résultats et d'engager des actions correspondantes, de suivre les réponses et de faire connaître les mesures prises pour parer aux incidences. La diligence raisonnable en matière de droits de l'homme peut être intégrée dans les systèmes généraux de gestion des risques de l'entreprise, à condition qu'ils ne se limitent pas à identifier et gérer les risques significatifs pour l'entreprise proprement dite, mais s'étendent aux risques pour les détenteurs de droits. Il s'agit d'une activité continue, étant entendu que les risques concernant les droits de l'homme peuvent changer à mesure que les activités de l'entreprise et son environnement évoluent. Les paragraphes A.10 à A.12 du chapitre sur les principes généraux et leurs commentaires formulent des indications complémentaires sur la diligence raisonnable, y compris en lien avec la chaîne d'approvisionnement, et des réponses adéquates aux risques liés aux chaînes d'approvisionnement.

46. Lorsqu'une entreprise détecte, grâce à son processus de diligence raisonnable ou par d'autres moyens, qu'elle a été la cause d'une incidence négative sur les droits de l'homme ou qu'elle y a contribué, 
les Principes directeurs recommandent de mettre en place des procédures pour y remédier. Dans certaines situations, il est nécessaire de coopérer avec des mécanismes judiciaires ou non judiciaires relevant de l'État. Dans d'autres cas, des mécanismes de réclamation au sein de l'entreprise disponibles pour les personnes susceptibles d'être affectées par les activités de l'entreprise peuvent être un moyen efficace d'établir de telles procédures, à condition de remplir les critères fondamentaux suivants : légitimité, accessibilité, prévisibilité, caractère équitable, compatibilité avec les Principes directeurs et transparence; ils doivent également être fondés sur le dialogue et la volonté de parvenir à des solutions concertées. Ces mécanismes peuvent être gérés par l'entreprise, seule ou en collaboration avec d'autres parties prenantes, et peuvent être source d'apprentissage continu. Les mécanismes de contestation au sein de l'entreprise ne devraient pas être utilisés en vue de saper le rôle des syndicats dans le règlement des conflits du travail, pas plus qu'ils ne devraient empêcher l'accès aux mécanismes de recours judiciaires ou non judiciaires, y compris aux Points de contact nationaux prévus par les Principes directeurs. 


\section{Emploi et relations professionnelles}

Les entreprises devraient, dans le cadre des lois et règlements applicables et des pratiques en vigueur en matière d'emploi et de relations du travail ainsi que des normes internationales du travail applicables

1. a) Respecter le droit des travailleurs employés par l'entreprise multinationale de constituer des syndicats et des organisations représentatives de leur choix ou de s'y affilier.

b) Respecter le droit des travailleurs employés par l'entreprise multinationale de mandater des syndicats et des organisations représentatives de leur choix afin de les représenter lors de négociations collectives, et d'engager, soit individuellement, soit par l'intermédiaire d'associations d'employeurs, des négociations constructives avec ces représentants, en vue d'aboutir à des accords sur les conditions d'emploi.

c) Contribuer à l'abolition effective du travail des enfants, et prendre des mesures immédiates et efficaces pour assurer de façon urgente l'interdiction et l'élimination des pires formes du travail des enfants.

d) Contribuer à l'élimination de toute forme de travail forcé ou obligatoire et prendre les mesures adéquates pour empêcher tout recours au travail forcé ou obligatoire dans leurs activités.

e) S'inspirer, dans leurs activités, du principe de l'égalité des chances et de traitement dans le travail, et ne pas pratiquer de discrimination envers leurs travailleurs en matière d'emploi ou de profession pour des motifs tels que la race, la couleur, le sexe, la religion, l'opinion politique, l'ascendance nationale ou l'origine sociale, ou toute autre circonstance, les pratiques sélectives concernant les caractéristiques des travailleurs ne pouvant que servir une politique établie des pouvoirs publics qui favorise spécifiquement une plus grande égalité des chances en matière d'emploi ou répondre aux exigences intrinsèques d'un emploi.

2. a) Fournir aux représentants des travailleurs les moyens nécessaires pour faciliter la mise au point de conventions collectives efficaces. 
b) Communiquer aux représentants des travailleurs les informations nécessaires à des négociations constructives sur les conditions d'emploi.

c) Fournir aux travailleurs et à leurs représentants les informations leur permettant de se faire une idée exacte et correcte de l'activité et des résultats de l'entité ou, le cas échéant, de l'entreprise dans son ensemble.

3. Promouvoir les consultations et la coopération entre les employeurs, les travailleurs et leurs représentants sur des sujets d'intérêt commun.

4. a) Observer en matière d'emploi et de relations du travail des normes aussi favorables que celles qui sont observées par des employeurs comparables dans le pays d'accueil.

b) Lorsque des entreprises multinationales opèrent dans des pays en développement où il peut ne pas exister des employeurs comparables, elles devraient octroyer les meilleurs salaires, prestations et conditions de travail possibles dans le cadre des politiques publiques. Ceux-ci devraient être en rapport avec la situation économique de l'entreprise, mais devraient être au moins suffisants pour satisfaire les besoins essentiels des travailleurs et de leurs familles.

c) Prendre les mesures nécessaires afin d'assurer dans leurs activités la santé et la sécurité du milieu de travail.

5. Dans leurs activités, dans toute la mesure du possible, employer du personnel local et assurer une formation en vue d'améliorer les niveaux de qualification, en coopération avec les représentants des travailleurs et, le cas échéant, avec les autorités publiques compétentes.

6. Lorsqu'elles envisagent d'apporter à leurs opérations des changements susceptibles d'avoir des effets importants sur les moyens d'existence de leurs travailleurs, notamment en cas de fermeture d'une entité entrainant des licenciements collectifs, en avertir dans un délai raisonnable les représentants de leurs travailleurs et, le cas échéant, les autorités nationales compétentes et coopérer avec ces représentants et autorités de façon à atténuer au maximum tout effet défavorable. Compte tenu des circonstances particulières dans chaque cas, il serait souhaitable que la direction en avertisse les intéressés avant que la décision définitive ne soit prise. D'autres moyens pourront être également utilisés pour que s'instaure une coopération constructive en vue d'atténuer les effets de telles décisions. 
7. Lors des négociations menées de bonne foi avec des représentants des travailleurs sur les conditions d'emploi, ou lorsque les travailleurs exercent leur droit de s'organiser, ne pas menacer de transférer hors du pays en cause tout ou partie d'une unité d'exploitation ni de transférer des travailleurs venant d'entités constitutives de l'entreprise situées dans d'autres pays en vue d'exercer une influence déloyale sur ces négociations ou de faire obstacle à l'exercice du droit de s'organiser.

8. Permettre aux représentants habilités de leurs travailleurs de mener des négociations sur les questions relatives aux conventions collectives ou aux relations entre salariés et employeurs et autoriser les parties à entreprendre des consultations sur les sujets d'intérêt commun avec les représentants patronaux habilités à prendre des décisions sur ces questions.

\section{Commentaire sur l'emploi et les relations professionnelles}

47. Ce chapitre débute par un chapeau qui fait référence aux lois et règlements «applicables» afin de tenir compte du fait que les entreprises multinationales, tout en opérant sur le territoire de certains pays, peuvent être soumises en matière d'emploi et de relations professionnelles à des dispositions nationales et internationales. Les termes «pratiques en vigueur en matière d'emploi et de relations de travail» sont suffisamment larges pour permettre diverses interprétations compte tenu des différentes situations nationales - par exemple, en ce qui concerne les différentes possibilités de négociation offertes aux travailleurs en vertu de la législation nationale.

48. L'Organisation internationale du travail (OIT) est l'organe compétent pour établir les normes internationales du travail et s'en occuper et pour promouvoir les droits fondamentaux au travail tels qu'ils sont reconnus dans la Déclaration de l'OIT de 1998 sur les principes et droits fondamentaux au travail. Les Principes directeurs, en tant qu'instrument non contraignant, ont un rôle à jouer pour promouvoir l'observation de ces normes et principes par les entreprises multinationales. Les Principes directeurs reflètent les dispositions pertinentes de la Déclaration de 1998 ainsi que de la Déclaration de principes tripartite de l'OIT de 1977 sur les entreprises multinationales et la politique sociale (révisée pour la dernière fois en 2006) (la « Déclaration de l'OIT sur les entreprises multinationales »). La Déclaration de l'OIT sur les entreprises multinationales établit des principes dans le domaine de l'emploi, de la formation, des conditions de travail et des relations professionnelles, tandis que les Principes directeurs couvrent tous les aspects majeurs de la conduite des entreprises. Les Principes directeurs 
de l'OCDE et la Déclaration de l'OIT sur les entreprises multinationales ont trait à la conduite attendue des entreprises, et s'inscrivent en parallèle et non en contradiction. La Déclaration de l'OIT sur les entreprises multinationales peut donc être utile pour la bonne compréhension des Principes directeurs en ce qu'elle est plus approfondie. Néanmoins, les procédures de suivi de la Déclaration de l'OIT sur les entreprises multinationales et des Principes directeurs ne relèvent pas des mêmes organes.

49. La terminologie employée dans le chapitre V est conforme à celle utilisée dans la Déclaration l'OIT sur les entreprises multinationales. Les termes «travailleurs employés par l'entreprise multinationale » et « leurs travailleurs » ont le même sens que dans la Déclaration de l'OIT sur les entreprises multinationales. Ils désignent les travailleurs qui sont «en relation de travail avec l'entreprise multinationale». Les entreprises qui souhaitent comprendre la portée de leur responsabilité au titre du chapitre $\mathrm{V}$ trouveront des orientations utiles pour déterminer l'existence d'une relation de travail dans le contexte des Principes directeurs dans la liste non exhaustive d'indices cités par la Recommandation 198 de l'OIT de 2006, paragraphes 13 (a) et (b). En outre, il est reconnu que les arrangements contractuels évoluent et se transforment au fil du temps et que les entreprises doivent structurer leurs relations avec leurs travailleurs de manière à éviter de promouvoir ou d'encourager des relations de travail déguisées ou d'y prendre part. Il y a relation de travail déguisée lorsqu'un employeur traite une personne autrement que comme un salarié d'une manière qui dissimule son statut juridique réel.

50. Ces recommandations n'entrent pas en conflit avec les relations civiles et commerciales proprement dites, mais cherchent plutôt à garantir que les personnes engagées dans une relation de travail bénéficient de la protection à laquelle elles ont droit au titre des Principes directeurs. Il est admis qu'en l'absence de relation de travail, les entreprises n'en sont pas moins tenues d'agir conformément aux recommandations relatives à la diligence raisonnable et à la chaîne d'approvisionnement, compte tenu des risques, qui figurent aux paragraphes A.10 à A.13 du chapitre II consacré aux Principes généraux.

51. Le premier paragraphe du présent chapitre fait référence aux quatre principes et droits fondamentaux au travail énoncés dans la Déclaration de l'OIT de 1998, à savoir la liberté d'association et le droit de négociation collective, l'abolition effective du travail des enfants, l'élimination de toute forme de travail forcé ou obligatoire et la nondiscrimination en matière d'emploi et de profession. Ces principes et droits ont été développés sous la forme de droits et obligations 
spécifiques dans les Conventions de l'OIT reconnues comme fondamentales.

52. Le paragraphe 1c) recommande que les entreprises multinationales contribuent à l'abolition effective du travail des enfants au sens de la Déclaration de l'OIT de 1998 et de la Convention 182 de l'OIT sur les pires formes de travail des enfants. La Convention 138 et la Recommandation 146 (adoptées en 1973), qui concernent l'âge minimum d'admission à l'emploi, sont deux instruments plus anciens de l'OIT dans le domaine du travail des enfants. Grâce à leurs pratiques en matière de gestion de la main-d'œuvre, aux emplois de haute qualité et bien rémunérés qu'elles créent et à leur contribution à la croissance économique, les entreprises multinationales peuvent jouer un rôle positif en aidant à s'attaquer aux causes fondamentales de la pauvreté en général et du travail des enfants en particulier. Il importe de reconnaître et de favoriser le rôle que peuvent jouer les entreprises multinationales en contribuant à la recherche d'une solution durable au problème du travail des enfants. À cet égard, l'amélioration du niveau d'éducation des enfants dans les pays d'accueil doit retenir tout particulièrement l'attention.

53. Le paragraphe 1d) recommande que les entreprises contribuent à l'élimination de toute forme de travail forcé et obligatoire, autre principe repris de la Déclaration de l'OIT de 1998. La référence à ce droit fondamental du travail se fonde sur les Conventions de l'OIT 29 (1930) et 105 (1957). La Convention 29 demande aux gouvernements de «supprimer l'emploi du travail forcé ou obligatoire sous toutes ses formes dans le plus bref délai possible» et la Convention 105 leur demande de «supprimer le travail forcé ou obligatoire et n'y recourir sous aucune forme » dans un certain nombre de cas énumérés (par exemple, en tant que moyen de coercition politique ou mesure de discipline du travail) et de "prendre des mesures efficaces en vue de l'abolition immédiate et complète du travail forcé ou obligatoire ». Dans le même temps, il est bien entendu que l'OIT est l'instance compétente en ce qui concerne la question délicate du travail carcéral, en particulier lorsqu'il s'agit de l'emploi de prisonniers par des particuliers, des entreprises ou des associations (ou leur mise à disposition).

54. La référence au principe de non-discrimination en matière d'emploi et de profession au paragraphe 1e s'applique aux conditions telles que l'embauche, l'affectation, le licenciement, la rémunération et autres avantages, l'avancement, le transfert ou la relocalisation, la cessation d'emploi, la formation et la retraite. La liste des formes interdites de discrimination énumérées dans la Convention 111 de l'OIT (1958), la Convention 183 sur la protection de la maternité (2000), la Convention 
159 sur la réadaptation professionnelle et l'emploi des personnes handicapées (1983), la Recommandation 162 sur les travailleurs âgés (1980) et la Recommandation 200 concernant le VIH et le SIDA et le monde du travail (2010), considère que toute distinction, exclusion ou préférence pour ces motifs est contraire aux Conventions, Recommandations et Codes. Le terme «autre circonstance» aux fins des Principes directeurs recouvre l'activité syndicale et des caractéristiques personnelles comme l'âge, l'invalidité, la grossesse, la situation de famille, l'orientation sexuelle et la sérologie VIH. Conformément aux dispositions du paragraphe 1e), les entreprises se doivent de promouvoir les mêmes opportunités pour les hommes et les femmes, en insistant tout particulièrement sur l'égalité des critères pour la sélection, la rémunération et l'avancement, et sur une application égalitaire de ces critères; elles se doivent également d'empêcher les discriminations ou le licenciement pour cause de mariage, de grossesse, de maternité ou de paternité.

55. Le paragraphe 2c) de ce chapitre dispose que les informations fournies par les entreprises aux travailleurs et à leurs représentants doivent donner une «idée exacte et correcte» des résultats. Ces informations concernent : la structure de l'entreprise, sa situation et ses perspectives économiques et financières, l'évolution de l'emploi et les changements importants attendus dans leurs activités, compte tenu des impératifs légitimes de confidentialité. L'élément de confidentialité signifie que les informations peuvent n'être pas fournies sur certains points, ou n'être fournies qu'avec certaines protections.

56. La référence aux formes consultatives de participation des travailleurs au paragraphe 3 du chapitre provient de la Recommandation 94 de l'OIT (1952) concernant la consultation et la collaboration entre employeurs et travailleurs sur le plan de l'entreprise. Elle est également conforme à une disposition de la Déclaration de l'OIT sur les entreprises multinationales. De tels dispositifs de consultation ne sauraient se substituer au droit des travailleurs de négocier leurs conditions de travail. Une recommandation au sujet des dispositifs de consultation relatifs aux conditions de travail figure également au paragraphe 8 .

57. Au paragraphe 4, les normes d'emploi et de relations professionnelles sont réputées couvrir la rémunération et le temps de travail. La référence à la santé et à la sécurité du milieu de travail implique que les entreprises multinationales se doivent d'appliquer les réglementations et les normes professionnelles en vigueur pour réduire le risque d'accident et de maladie professionnelle en cours d'emploi ou en liaison avec l'emploi. Il s'agit d'encourager les entreprises à travailler à l'amélioration du niveau de performance en matière de santé et de 
sécurité du milieu du travail dans toutes leurs composantes, même si ceci n'est pas formellement exigé par les réglementations en vigueur dans les pays où elles opèrent. Il s'agit aussi d'encourager les entreprises à respecter la possibilité, pour les travailleurs, de se retirer d'une situation de travail lorsqu'il y a des motifs raisonnables de penser qu'elle présente un danger imminent et grave pour la santé ou la sécurité. Les problèmes de santé et de sécurité, vu leur importance et les complémentarités avec d'autres recommandations, sont traités également dans d'autres dispositions des Principes directeurs, surtout à propos de la protection des consommateurs et de l'environnement. La Recommandation 194 de l'OIT (2002) fournit une liste indicative des maladies professionnelles et précise les recueils de directives pratiques ou guides que les entreprises doivent prendre en compte pour mettre en œuvre cette recommandation des Principes directeurs.

58. En vertu de la recommandation énoncée au paragraphe 5, les entreprises multinationales sont invitées à recruter sur place une proportion adéquate de leur main-d'œuvre (y compris le personnel de gestion) et à leur assurer une formation. Les dispositions de ce paragraphe concernant la formation et le niveau de qualification complètent le texte du paragraphe A. 4 du chapitre sur les principes généraux qui a trait à la promotion de la formation du capital humain. La référence au personnel local complète le texte encourageant la création de capacités locales au paragraphe A. 3 du chapitre sur les principes généraux. Conformément à la Recommandation 195 de l'OIT sur la mise en valeur des ressources humaines (2004), les entreprises sont également encouragées à investir, dans toute la mesure du possible, dans l'éducation et la formation tout au long de la vie, tout en garantissant l'égalité des chances dans l'éducation pour les femmes et d'autres catégories vulnérables, telles que les jeunes, les personnes peu qualifiées, les personnes handicapées, les migrants, les travailleurs âgés et les populations autochtones.

59. Le paragraphe 6 recommande aux entreprises d'avertir dans un délai raisonnable les représentants des travailleurs et les autorités publiques compétentes lorsqu'elles envisagent d'apporter à leurs opérations des changements susceptibles d'avoir des effets importants sur les moyens d'existence de leurs travailleurs, notamment en cas de fermeture d'une entité entraînant des licenciements collectifs. Comme il est indiqué dans ce paragraphe, le but est de ménager la possibilité d'une coopération afin d'atténuer les effets de ces changements. C'est là un principe important qui se reflète largement dans les réglementations et pratiques relatives aux relations du travail des pays souscrivant aux Principes directeurs, même si les solutions adoptées pour ménager la possibilité d'une coopération constructive ne sont pas identiques dans tous les pays 
considérés. Comme l'indique également ce paragraphe, il conviendrait, compte tenu des circonstances particulières dans chaque cas, que la direction en avertisse les intéressés avant que la décision définitive soit prise. De fait, un tel préavis avant la décision finale est prévu dans les réglementations et pratiques relatives aux relations du travail d'un certain nombre de pays souscrivant aux Principes directeurs. Mais ce n'est pas le seul moyen de ménager la possibilité d'une coopération constructive pour atténuer les effets de ce type de décisions, et les lois et pratiques d'autres pays souscrivant aux Principes directeurs prévoient d'autres moyens en fixant notamment un délai au cours duquel des consultations doivent avoir lieu avant qu'une décision puisse être mise en œuvre. 


\section{Environnement}

Les entreprises devraient, dans le cadre des lois, règlements et pratiques administratives en vigueur dans les pays où elles opèrent, et eu égard aux accords, principes, objectifs et normes internationaux pertinents, tenir dûment compte de la nécessité de protéger l'environnement, la santé et la sécurité publiques, et d'une manière générale, de conduire leurs activités d'une manière qui contribue à l'objectif plus large de développement durable. En particulier, les entreprises devraient :

1. Mettre en place et appliquer un système de gestion environnementale adapté à l'entreprise et prévoyant :

a) la collecte et l'évaluation en temps utile d'informations adéquates relatives aux effets potentiels de leurs activités sur l'environnement, la santé et la sécurité ;

b) la fixation d'objectifs mesurables et, en tant que de besoin, spécifiques concernant l'amélioration de leurs performances environnementales et de l'utilisation de leurs ressources, et un examen périodique de la pertinence de ces objectifs ; le cas échéant, les objectifs devraient être cohérents avec les politiques nationales et les engagements internationaux pertinents; et

c) le suivi et le contrôle réguliers des progrès réalisés dans la poursuite des objectifs généraux et spécifiques en matière d'environnement, de santé et de sécurité.

2. Eu égard aux considérations liées aux coûts, à la confidentialité des affaires et aux droits de propriété intellectuelle :

a) fournir au public et aux travailleurs en temps voulu des informations adéquates, mesurables et vérifiables (si possible) relatives aux effets potentiels de leurs activités sur l'environnement, la santé et la sécurité, ces informations pouvant comprendre un bilan des progrès accomplis dans l'amélioration des performances environnementales; et 
b) entrer en temps voulu en communication et en consultation avec les collectivités directement concernées par les politiques de l'entreprise en matière d'environnement, de santé et de sécurité et par leur mise en œuvre.

3. Évaluer et prendre en compte, lors de la prise de décision, les effets prévisibles sur l'environnement, la santé et la sécurité, des procédés, biens et services de l'entreprise sur l'ensemble de leur cycle de vie en vue d'éviter ces effets et, s'ils sont inévitables, de les atténuer. Lorsque les activités envisagées risquent d'avoir des effets importants sur l'environnement, la santé ou la sécurité, et qu'elles sont subordonnées à une décision d'une autorité compétente, les entreprises devraient réaliser une évaluation appropriée d'impact sur l'environnement.

4. Compte tenu des connaissances scientifiques et techniques des risques, lorsqu'il existe des menaces de dommages graves pour l'environnement, compte tenu également de la santé et la sécurité humaines, ne pas invoquer l'absence de certitude scientifique absolue pour remettre à plus tard l'adoption de mesures efficaces par rapport aux coûts destinées à prévenir ou réduire ces dommages.

5. Établir des plans d'urgence afin de prévenir, d'atténuer et de maîtriser les dommages graves à l'environnement et à la santé pouvant résulter de leurs activités, y compris du fait d'accidents et de situations d'urgence, et mettre en place des mécanismes d'alerte immédiate des autorités compétentes.

6. S'efforcer constamment d'améliorer leurs performances environnementales au niveau de l'entreprise et, le cas échéant, de sa chaîne d'approvisionnement, en encourageant des activités telles que :

a) l'adoption, dans toutes les composantes de l'entreprise, de technologies et de procédures d'exploitation qui reflètent les normes de performance environnementale de la composante la plus performante de l'entreprise ;

b) la mise au point et la fourniture de produits ou de services qui n'ont pas d'incidences indues sur l'environnement, dont l'utilisation aux fins prévues est sans danger, qui réduisent les émissions de gaz à effet de serre, qui sont économes en énergie et en ressources naturelles, et qui peuvent être réutilisés, recyclés ou éliminés en toute sécurité ;

c) la sensibilisation de leurs clients aux conséquences environnementales de l'utilisation des produits et services de 
l'entreprise, en fournissant des informations exactes sur leurs produits (par exemple sur les émissions de gaz à effet de serre, la biodiversité, l'utilisation efficiente des ressources ou d'autres aspects environnementaux); et

d) l'étude et l'évaluation des moyens d'améliorer à long terme les performances environnementales de l'entreprise, par exemple en élaborant des stratégies de réduction des émissions, d'utilisation efficiente des ressources, de recyclage, de remplacement ou de réduction de l'utilisation de substances toxiques, ou des stratégies portant sur la biodiversité.

7. Offrir aux travailleurs un enseignement et une formation appropriés sur les questions de santé et de sécurité de l'environnement, notamment la manipulation des matières dangereuses et la prévention des accidents affectant l'environnement, ainsi que sur les aspects plus généraux de la gestion environnementale, tels que les procédures d'évaluation d'impact sur l'environnement, les relations publiques et les technologies environnementales.

8. Contribuer à la mise au point d'une politique publique en matière d'environnement qui soit bien conçue et économiquement efficiente au moyen, par exemple, de partenariats ou d'initiatives susceptibles d'améliorer la sensibilisation et la protection environnementales.

\section{Commentaire sur l'environnement}

60. Le chapitre sur l'environnement reflète largement les principes et objectifs énoncés dans la Déclaration de Rio sur l'environnement et le développement, dans Action 21 (dans le cadre de la Déclaration de Rio) et dans la Convention (d'Aarhus) sur l'accès à l'information, la participation du public au processus décisionnel et l'accès à la justice en matière d'environnement. Il reflète également les normes édictées dans des instruments comme la norme ISO sur les systèmes de gestion environnementale.

61. Une saine gestion de l'environnement est un volet essentiel du développement durable et elle est considérée de plus en plus comme une responsabilité et une opportunité pour les entreprises. Les entreprises multinationales ont un rôle à jouer sur ces deux plans. Il convient donc que les dirigeants de ces entreprises prêtent l'attention qui s'impose aux problèmes environnementaux dans leurs stratégies. L'amélioration des performances environnementales nécessite un engagement envers une approche systématique et une amélioration continue du système. Un 
système de gestion environnementale offre le cadre interne nécessaire pour contrôler l'impact environnemental d'une entreprise et pour intégrer les considérations environnementales dans ses activités. La mise en place d'un tel système devrait contribuer à assurer les actionnaires, les salariés et la communauté de la volonté de l'entreprise de préserver l'environnement de l'impact de ses activités.

62. Outre l'amélioration des performances environnementales, la mise en place d'un système de gestion environnementale peut présenter des avantages économiques pour les entreprises grâce à des coûts réduits d'exploitation et d'assurance, des économies d'énergie et de ressources, des charges plus faibles de conformité et de responsabilité, un accès plus facile au capital et aux compétences, une plus grande satisfaction des clients et de meilleures relations avec la communauté et le public.

63. Dans le contexte des Principes directeurs, l'expression «système de gestion environnementale adapté » doit être interprétée très largement, en couvrant les activités de l'entreprise qui visent à maîtriser l'impact direct et indirect à long terme sur l'environnement, la lutte contre la pollution et la gestion des ressources.

64. Dans la plupart des entreprises, un système de contrôle interne est nécessaire à la gestion des activités de l'entreprise. Les composantes environnementales de ce système peuvent comprendre des éléments tels que des objectifs de meilleure performance et des contrôles réguliers des progrès accomplis dans la réalisation de ces objectifs.

65. Les informations concernant les activités des entreprises ainsi que leurs relations avec leurs sous-traitants et fournisseurs et l'impact environnemental qui y est lié contribuent à instaurer un climat de confiance avec le public. Ceci est particulièrement efficace lorsque les informations sont fournies de manière transparente et qu'on encourage des consultations actives avec les parties prenantes telles que les salariés, les clients, les fournisseurs, les contractants, les communautés locales et le public en général, afin de promouvoir une atmosphère de confiance et de compréhension à long terme sur les questions environnementales d'intérêt commun. Les rapports et la communication sont particulièrement appropriés lorsque des biens environnementaux rares ou à risque sont en jeu, dans un contexte régional, national ou international ; des normes de reporting telles que la Global Reporting Initiative fournissent des références utiles.

66. Pour fournir des informations exactes sur leurs produits, les entreprises ont à leur disposition diverses solutions, telles que des mécanismes volontaires d'étiquetage et de certification. Lorsqu'elles y recourent, elles devraient tenir dûment compte de leurs répercussions économiques 
et sociales sur les pays en développement, ainsi que de l'existence de normes reconnues à l'échelle internationale.

67. L'activité normale de l'entreprise peut impliquer une évaluation a priori de l'impact potentiel de ses opérations sur l'environnement. Les entreprises procèdent souvent à des évaluations d'impact environnemental appropriées, même si la loi ne les y oblige pas. Les évaluations environnementales effectuées par les entreprises peuvent mesurer, dans une optique large et prospective, l'impact potentiel de leurs activités et de celles des sous-traitants et des fournisseurs, en prenant en compte les effets pertinents et en examinant d'autres options et des mesures d'atténuation afin d'éviter les effets négatifs ou d'y remédier. Les Principes directeurs reconnaissent également que les entreprises multinationales ont certaines responsabilités à d'autres stades du cycle de vie du produit.

68. Plusieurs instruments adoptés par les pays souscrivant aux Principes directeurs, notamment le principe 15 de la Déclaration de Rio sur l'environnement et le développement, se réfèrent au «principe de précaution ». Aucun de ces instruments ne s'adresse expressément aux entreprises, bien que la contribution des entreprises soit implicite dans la totalité d'entre eux.

69. Les Principes directeurs posent en prémisse que les entreprises devraient prendre des mesures le plus tôt possible et de façon anticipative pour éviter, par exemple, les dommages graves ou irréversibles à l'environnement liés à leurs activités. Toutefois, le fait que les Principes directeurs s'adressent aux entreprises signifie qu'aucun instrument actuel n'est totalement adéquat pour exprimer cette recommandation. En conséquence, les Principes directeurs s'appuient sur les instruments existants, mais ne les reflètent pas totalement.

70. Les Principes directeurs n'ont pas pour but de réinterpréter les instruments actuels ni de créer, pour les pouvoirs publics, de nouveaux engagements ou des précédents; ils ont uniquement pour but de recommander comment mettre en œuvre une approche de précaution au niveau des entreprises. Ce processus n'en étant qu'à ses débuts, on reconnaît que son application nécessite une certaine souplesse, en fonction du contexte spécifique dans lequel il intervient. On reconnaît également que les pouvoirs publics déterminent dans ce domaine le cadre de base et qu'il leur incombe de consulter périodiquement les parties prenantes sur la meilleure façon d'aller de l'avant.

71. Les Principes directeurs encouragent également les entreprises à s'efforcer d'améliorer le niveau de performance environnementale de toutes leurs composantes, même si les pays dans lesquels elles opèrent 
ne l'exigent pas expressément dans la pratique. A cet égard, les entreprises devraient prendre dûment en compte leur impact social et économique sur les pays en développement.

72. Par exemple, les entreprises multinationales sont souvent à même de faire appel à des technologies existantes ou innovantes ou à des procédures opérationnelles qui pourraient, si elles étaient utilisées, contribuer à améliorer globalement les performances environnementales. Les entreprises multinationales étant fréquemment considérées comme des chefs de file dans leur secteur, il ne faut pas oublier qu'elles peuvent exercer un effet d'émulation sur les autres entreprises. Faire en sorte que l'environnement des pays dans lesquels les entreprises multinationales opèrent bénéficie également des technologies et des pratiques disponibles et innovantes est un moyen important de soutenir les activités d'investissement international sur un plan plus général.

73. Les entreprises ont un grand rôle à jouer dans la formation et l'éducation de leurs salariés en matière d'environnement. Elles sont encouragées à s'acquitter de cette responsabilité de manière aussi large que possible, particulièrement dans les domaines touchant directement à la santé et la sécurité humaines. 


\section{Lutte contre la corruption, la sollicitation de pots-de-vin et d'autres formes d'extorsion}

Les entreprises ne devraient pas, directement ou indirectement, offrir, promettre, accorder ou exiger des paiements illicites ou d'autres avantages indus en vue d'obtenir ou de conserver un marché ou un autre avantage illégitime. Les entreprises devraient également repousser toute sollicitation de pots-de-vin et autres formes d'extorsion. En particulier, les entreprises :

1. Ne devraient pas offrir, promettre ou accorder des avantages indus, pécuniaires ou autres, à des agents publics ou à des salariés de leurs partenaires commerciaux. De la même façon, elles ne devraient pas demander, convenir de recevoir ou accepter des avantages indus, pécuniaires ou autres, d'agents publics ou de salariés de leurs partenaires commerciaux. Les entreprises ne devraient pas avoir recours à des tiers tels que des agents ou autres intermédiaires, consultants, représentants, distributeurs, consortiums, contractants et fournisseurs ou associés dans des co-entreprises pour faire parvenir des avantages indus, pécuniaires ou autres, à des agents publics ou à des salariés de leurs partenaires commerciaux, ou à des membres de la famille ou associés de ceux-ci.

2. Devraient mettre au point et adopter des mécanismes de contrôle interne et des programmes ou des mesures de déontologie et de conformité appropriés visant à prévenir et à détecter la corruption, élaborés à partir d'une évaluation des risques prenant en compte les circonstances individuelles propres à chaque entreprise, en particulier des risques de corruption auxquels elle pourrait être confrontée (par exemple son implantation géographique ou son secteur d'activité). Ces mécanismes de contrôle interne et programmes ou mesures de déontologie et de conformité devraient comprendre notamment un ensemble de procédures financières et comptables, en particulier un système de contrôles internes conçu de manière à donner une assurance raisonnable sur la tenue de livres, registres et comptes sincères et exacts permettant de garantir qu'ils ne pourront pas être utilisés à des fins de corruption ou de dissimulation d'actes de corruption. Ces circonstances individuelles et risques de corruption devraient être régulièrement surveillés et 
réévalués en tant que de besoin pour garantir que les mécanismes de contrôle interne et les programmes ou mesures de déontologie et de conformité adoptés par l'entreprise sont bien adaptés et restent efficaces, et réduire le risque qu'elle se rende complice d'actes de corruption, de sollicitation de pots-de-vin ou d'autres formes d'extorsion.

3. Devraient insérer, dans leurs mécanismes de contrôle interne et dans leurs programmes ou mesures de déontologie et de conformité, des dispositions interdisant ou dissuadant de recourir à de petits paiements de facilitation, qui sont généralement illégaux dans les pays où ils sont effectués et, si de tels paiements existent, les comptabiliser précisément dans les livres et états financiers.

4. Devraient, en tenant compte des risques particuliers de corruption auxquels elles sont confrontées, exercer une diligence raisonnable, attestée par des documents en bonne et due forme, vis-à-vis du recrutement d'agents, ainsi que de leur contrôle régulier et approprié, et s'assurer que la rémunération de ces agents est correcte et n'est versée que pour des services légitimes. Le cas échéant, une liste des agents engagés dans le cadre de transactions avec des organismes publics ou des entreprises publiques devrait être établie et tenue à la disposition des autorités compétentes, conformément aux obligations applicables en matière de communication d'informations au public.

5. Devraient améliorer la transparence de leurs activités de lutte contre la corruption, la sollicitation de pots-de-vin et les autres formes d'extorsion. Elles pourraient ainsi par exemple prendre des engagements publics contre la corruption, la sollicitation de pots-de-vin et les autres formes d'extorsion, et communiquer des informations sur les systèmes de gestion et sur les mécanismes de contrôle interne ou les programmes et mesures de déontologie et de discipline adoptés par elles afin de tenir ces engagements. Les entreprises devraient également encourager l'ouverture et le dialogue avec le public afin de le sensibiliser à la question de la lutte contre la corruption, la sollicitation de pots-de-vin et d' autres formes d'extorsion et de s'assurer sa coopération.

6. Devraient informer leurs salariés de leurs politiques et de leurs mécanismes de contrôle interne ainsi que de leurs programmes ou mesures de déontologie et de conformité ou des mesures qu'elles prennent pour lutter contre la corruption, la sollicitation de pots-de-vin et les autres formes d'extorsion, et promouvoir le respect de ces dispositions par les salariés, en assurant une diffusion convenable de ces politiques, programmes ou mesures et en mettant en place des programmes de formation et des procédures disciplinaires. 
7. Ne devraient verser aucune contribution illégale à des candidats à des charges publiques, à des partis politiques ou à d'autres organisations politiques. Les contributions politiques devraient se conformer intégralement aux règles de publication d'informations et être portées à la connaissance des dirigeants de l'entreprise.

\section{Commentaire sur la lutte contre la corruption, la sollicitation de pots- de-vin et d'autres formes d'extorsion}

74. La corruption, active ou passive, est préjudiciable aux institutions démocratiques et au gouvernement d'entreprise. Elle décourage l'investissement et exerce des distorsions sur la compétitivité internationale. En particulier, les détournements de fonds dus à la corruption sapent l'action menée par les citoyens pour améliorer leur bien-être économique, social et environnemental et entravent en outre les efforts de réduction de la pauvreté. Les entreprises ont un grand rôle à jouer dans la lutte contre de telles pratiques.

75. Légitimé, intégrité et transparence dans le domaine public comme dans le domaine privé sont des valeurs essentielles de la lutte contre la corruption et les diverses formes d'extorsion. La communauté des affaires, les organisations non gouvernementales, les pouvoirs publics et les organisations intergouvernementales coopèrent pour renforcer le soutien du public envers les mesures de lutte contre la corruption et pour améliorer la transparence et la sensibilisation du public aux problèmes soulevés par la corruption active et passive. L'adoption de pratiques adéquates de gouvernement d'entreprise constitue également un volet essentiel pour favoriser une culture de l'éthique au sein de l'entreprise.

76. La Convention de l'OCDE sur la lutte contre la corruption d'agents publics étrangers dans les transactions commerciales internationales (la «Convention sur la lutte contre la corruption ») est entrée en vigueur le 15 février 1999. La Convention sur la lutte contre la corruption, de même que la Recommandation de 2009 visant à renforcer la lutte contre la corruption d'agents publics étrangers dans les transactions commerciales internationales (la «Recommandation de $2009 »$ ), la Recommandation de 2009 sur les mesures fiscales visant à renforcer la lutte contre la corruption d'agents publics étrangers dans les transactions commerciales internationales et la Recommandation de 2006 sur la corruption et les crédits à l'exportation bénéficiant d'un soutien public, sont les principaux instruments de l'OCDE visant les activités de corruption du côté de l'offre. Ils ont pour but d'éliminer l'« offre » de pots-de-vin à des agents publics étrangers, chaque pays étant compétent pour les activités de ses entreprises et les actes commis sur 
son territoire. ${ }^{6}$ Un dispositif de suivi rigoureux et systématique de la mise en œuvre, par les pays, de la Convention sur la lutte contre la corruption afin de favoriser la pleine application de ces instruments.

77. Dans la Recommandation de 2009 en particulier, les signataires sont invités à encourager leurs entreprises à mettre au point et à adopter des mécanismes de contrôle interne et des programmes ou mesures de déontologie et de discipline adéquats afin de prévenir et de détecter les actes de corruption transnationale, en se fondant sur le Guide de bonnes pratiques pour les contrôles internes, la déontologie et la conformité qui forme l'annexe II de la Recommandation de 2009. Ce Guide de bonnes pratiques, qui s'adresse aux entreprises aussi bien qu'aux organisations patronales et professionnelles, met en exergue les bonnes pratiques permettant de garantir l'efficacité des mécanismes de contrôle interne et des programmes et mesures de déontologie et de conformité destinés à prévenir et à détecter les actes de corruption transnationale.

78. Des initiatives émanant du secteur privé et de la société civile peuvent également aider les entreprises à concevoir et à appliquer des stratégies efficaces de lutte contre la corruption.

79. La Convention des Nations Unies contre la corruption, qui est entrée en vigueur le 14 décembre 2009, contient un large éventail de normes, de mesures et de règles destinées à lutter contre la corruption. Les États parties à cette Convention des Nations Unies doivent interdire à leurs agents publics de recevoir des pots-de-vin et à leurs entreprises de verser des pots-de-vin à des agents publics de leur pays, ainsi qu'à des agents publics étrangers ou à des agents appartenant à des organisations internationales publiques, et envisager par ailleurs de refuser la corruption entre acteurs du secteur privé. La Convention des Nations

6.

Dans la Convention, un «pot-de-vin» est défini comme le fait «d'offrir, de promettre ou d'octroyer un avantage indu pécuniaire ou autre, directement ou par des intermédiaires, à un agent public étranger, à son profit ou au profit d'un tiers, pour que cet agent agisse ou s'abstienne d'agir dans l'exécution de fonctions officielles, en vue d'obtenir ou conserver un marché ou un autre avantage indu dans le commerce international. » Dans les Commentaires relatifs à la Convention, il est précisé (au paragraphe 9) que «Les petits paiements dits de «facilitation » ne constituent pas des paiements « en vue d'obtenir ou de conserver un marché ou un autre avantage indu » au sens du paragraphe 1 et, en conséquence, ils ne constituent pas une infraction au sens de cette disposition. De tels paiements, qui sont faits, dans certains pays, pour inciter les agents publics à exécuter leurs fonctions, notamment lorsqu'il s'agit de délivrer une autorisation ou un permis, sont généralement illicites dans le pays étranger concerné. Les autres pays peuvent et devraient s'attaquer à ce phénomène corrosif par des mesures telles que le soutien de programmes de bonne gestion des affaires publiques. ». 
Unies et la Convention de l'OCDE sur la lutte contre la corruption se renforcent mutuellement et sont complémentaires.

80. Sous l'angle de la «demande », de saines pratiques de gouvernement d'entreprise sont importantes en ce qu'elles contribuent à éviter aux entreprises d'être confrontées à des sollicitations de pots-de-vin. Les entreprises peuvent soutenir les initiatives collectives visant à résister à la sollicitation de pots-de-vin et à d'autres formes d'extorsion. Leurs gouvernements comme les pouvoirs publics des pays d'accueil devraient aider les entreprises confrontées à des sollicitations de potsde-vin ou à des tentatives d'extorsion. Le Guide de bonnes pratiques sur certains articles de la Convention qui figure en annexe I de la Recommandation de 2009 dispose que la Convention sur la lutte contre la corruption devrait être mise en œuvre de façon à ne pas prévoir de moyen de défense ou d'exception dans les cas où un agent public étranger sollicite un pot-de-vin. Par ailleurs, la Convention des Nations Unies prévoit d'attribuer la qualification pénale à la sollicitation de pots-de-vin par des agents publics nationaux. 


\section{Intérêts des consommateurs}

Dans leurs relations avec les consommateurs, les entreprises devraient se conformer à des pratiques loyales dans l'exercice de leurs activités en matière commerciale, de marketing et de publicité et prendre toutes les mesures raisonnables pour garantir la qualité et la fiabilité des biens ou des services qu'elles fournissent. En particulier, elles devraient :

1. Veiller à ce que les biens et les services qu'elles fournissent soient conformes à toutes les normes requises en matière de santé et de sécurité des consommateurs, notamment à celles concernant les mises en garde en matière de santé et les informations sur la sécurité.

2. Donner des renseignements exacts, vérifiables et clairs qui soient suffisants pour permettre aux consommateurs de prendre leurs décisions en connaissance de cause, notamment des renseignements sur les prix et, s'il y a lieu, le contenu, la sécurité d'utilisation, les effets sur l'environnement, l'entretien, le stockage et l'élimination des biens et des services. Si possible, ces informations devraient être formulées de manière à permettre aux consommateurs de comparer les produits.

3. Fournir aux consommateurs l'accès à des mécanismes extrajudiciaires de règlement des différends et des mesures correctrices équitables, faciles à utiliser, rapides et efficaces sans coûts ou charges inutiles.

4. S'abstenir de toute affirmation, omission ou toute autre pratique qui soit trompeuse, fallacieuse, frauduleuse ou déloyale.

5. Soutenir les efforts en vue de promouvoir l'éducation des consommateurs dans les domaines qui relèvent de leurs activités commerciales, notamment dans le but d'améliorer la capacité des consommateurs : $i$ ) de prendre des décisions en connaissance de cause concernant des biens, des services et des marchés complexes, ii) de mieux comprendre l'impact économique, environnemental et social de leurs décisions et iii) de soutenir la consommation durable. 
6. Respecter la vie privée des consommateurs et prendre des mesures raisonnables pour assurer la sécurité des données à caractère personnel qu'elles collectent, conservent, traitent ou diffusent.

7. Coopérer étroitement avec les autorités publiques pour empêcher et combattre les pratiques commerciales mensongères (y compris la publicité trompeuse et la fraude commerciale) et réduire ou empêcher les menaces graves à la santé et à la sécurité publiques ou à l'environnement résultant de la consommation, de l'utilisation ou de l'élimination de leurs biens et de leurs services.

8. Prendre en considération, dans l'application des principes ci-dessus, i) les besoins des consommateurs vulnérables et défavorisés et ii) les problèmes spécifiques que le commerce électronique peut poser aux consommateurs.

\section{Commentaire sur les intérêts des consommateurs}

81. Le chapitre des Principes directeurs de l'OCDE à l'intention des entreprises multinationales sur les intérêts des consommateurs s'appuie sur les travaux du Comité de la politique à l'égard des consommateurs et du Comité des marchés financiers de l'OCDE ainsi que sur les travaux d'autres organisations internationales, notamment la Chambre de commerce internationale, l'Organisation internationale de normalisation et les Nations Unies (c'est-à-dire les Principes directeurs des Nations Unies pour la protection du consommateur tels qu'étendus en 1999).

82. Le chapitre reconnaît que la satisfaction du consommateur et la défense de ses intérêts constituent une base essentielle pour un fonctionnement satisfaisant des entreprises. Il reconnaît par ailleurs que les marchés de biens et de services de consommation ont subi une transformation majeure au fil du temps. Les réformes de la réglementation, l'ouverture des marchés au niveau mondial, le développement des nouvelles technologies et le développement des services aux consommateurs ont constitué des éléments essentiels du changement, en offrant aux consommateurs davantage de choix ainsi que les autres bénéfices qui résultent d'une plus grande ouverture à la concurrence. Par ailleurs, du fait du rythme du changement et de la complexité accrue de nombreux marchés, il est devenu en général plus difficile aux consommateurs de comparer et d'évaluer les produits. De plus, la démographie des consommateurs s'est également modifiée au fil du temps. Les enfants deviennent de plus en plus des forces importantes sur le marché, et il en 
est de même des adultes âgés qui sont de plus en plus nombreux. Bien que les consommateurs soient dans l'ensemble mieux formés, il manque encore à un grand nombre d'entre eux les compétences en arithmétique et l'aptitude à la lecture qui sont requises désormais sur des marchés de plus en plus complexes et à forte intensité d'information. En outre, beaucoup de consommateurs cherchent de plus en plus à connaître la position et les activités des entreprises dans un large éventail de domaines économiques, sociaux et environnementaux et prennent ces considérations en compte lorsqu'elles choisissent des produits.

83. Le chapeau appelle les entreprises à recourir à des pratiques loyales en matière commerciale, de distribution et de publicité et à s'assurer de la qualité et de la fiabilité des produits qu'elles fournissent. Il y a lieu de noter que ces principes s'appliquent à la fois aux biens et aux services.

84. Le paragraphe 1 souligne la nécessité, pour les entreprises, d'adhérer aux normes requises en matière de santé et de sécurité ainsi que de donner aux consommateurs des informations suffisantes concernant les effets de leurs produits sur la santé et la sécurité.

85. Le paragraphe 2 concerne la divulgation d'informations. Il appelle les entreprises à fournir des informations qui soient suffisantes pour que les consommateurs puissent prendre leurs décisions en connaissance de cause. Il s'agirait notamment, le cas échéant, d'informations sur les risques financiers associés aux produits. Par ailleurs, les entreprises sont parfois légalement tenues de fournir des informations qui permettent d'effectuer des comparaisons directes entre produits (prix unitaire, par exemple). En l'absence de législation contraignante, les entreprises sont incitées, dans leurs relations avec les consommateurs, à fournir des informations qui facilitent les comparaisons entre produits et qui permettent de déterminer le coût total d'un produit. Il y a lieu de noter que ce qui est considéré comme « suffisant » peut varier au fil du temps et que les entreprises devraient réagir à ces changements. Toutes les déclarations faites par les entreprises en ce qui concerne les produits et l'environnement devraient être fondées sur des éléments de preuve suffisants et, s'il y a lieu, des tests appropriés. Étant donné l'intérêt croissant des consommateurs pour les questions d'environnement et la consommation durable, des informations devraient être données, le cas échéant, au sujet de l'impact des produits sur l'environnement. Il pourrait s'agir d'informations sur l'efficience énergétique et le degré de recyclabilité des produits, et, dans le cas des produits alimentaires, d'informations sur les procédés agricoles.

86. Les consommateurs tiennent de plus en plus compte de la conduite des entreprises lorsqu'ils prennent leurs décisions d'achat. Les entreprises 
sont par conséquent invitées à communiquer des informations sur les initiatives qu'elles ont prises pour intégrer les préoccupations sociales et environnementales dans leurs activités industrielles ou commerciales et pour soutenir par ailleurs la consommation durable. Le chapitre III des Principes directeurs sur la publication d'informations est significatif à cet égard. Les entreprises y sont invitées à publier des déclarations sur leurs valeurs ou leurs pratiques commerciales, comprenant des informations sur leurs politiques sociales, éthiques et environnementales et autres codes de conduite auxquelles elles adhèrent. Les entreprises sont invitées à rendre ces informations disponibles en langage simple et sous une forme qui soit attrayante pour les consommateurs. Il serait souhaitable qu'un nombre croissant d'entreprises communiquent dans ces domaines et ciblent les informations sur les consommateurs.

87. Le paragraphe 3 reflète les termes utilisés dans la Recommandation de 2007 du Conseil sur le règlement des litiges de consommation et leur réparation. La Recommandation fixe un cadre pour la mise au point de méthodes efficaces de traitement des réclamations des consommateurs, et notamment une série de mesures que les différents secteurs peuvent prendre à cet égard. Il est fait observer que les mécanismes mis en place par un grand nombre d'entreprises pour résoudre les différends en matière de consommation ont contribué à renforcer la confiance et la satisfaction des consommateurs. Ces mécanismes peuvent déboucher en cas de réclamation sur des solutions plus facilement applicables que les actions judiciaires, qui peuvent être complexes et coûteuses en temps et en argent pour toutes les parties concernées. Cependant, pour que ces mécanismes extrajudiciaires soient efficaces, il faut que les consommateurs soient au courant de leur existence et il serait souhaitable de leur communiquer des indications sur les moyens de déposer des réclamations, notamment lorsque leurs demandes portent sur des transactions transfrontalières ou multidimensionnelles.

88. Le paragraphe 4 porte sur les pratiques commerciales trompeuses, fallacieuses, frauduleuses et autres pratiques déloyales. Ces pratiques peuvent provoquer des distorsions sur les marchés, aux dépens des consommateurs et des entreprises responsables et il y a lieu de les éviter.

89. Le paragraphe 5 concerne l'éducation des consommateurs, qui a pris une grande importance avec la complexité croissante d'un grand nombre de marchés et de produits. Les gouvernements, les organisations de consommateurs et beaucoup d'entreprises ont reconnu qu'il s'agit d'une responsabilité partagée et qu'ils peuvent jouer des rôles importants à cet égard. Les difficultés que les consommateurs ont connues dans l'évaluation des produits complexes, notamment dans le domaine 
financier, ont souligné la nécessité pour les différentes parties prenantes de travailler ensemble en vue de promouvoir une éducation visant à améliorer la prise de décisions par les consommateurs.

90. Le paragraphe 6 concerne les données de caractère personnel. Le développement de la collecte et de l'utilisation de données à caractère personnel par les entreprises, alimenté en partie par l'Internet et les avancées technologiques, a mis en lumière la nécessité de préserver ces données contre toute violation de la vie privée des consommateurs, y compris contre les menaces à leur sécurité.

91. Le paragraphe 7 souligne la nécessité, pour les entreprises, de coopérer avec les autorités publiques pour contribuer à empêcher et à combattre les pratiques commerciales mensongères d'une manière plus efficace. Elles sont également appelées à coopérer pour réduire ou empêcher les menaces pesant sur la santé et la sécurité publiques ainsi que sur l'environnement. Cela inclut aussi bien les menaces qui sont liées à l'élimination des produits que celles qui portent sur leur consommation et leur utilisation. Cela traduit la prise de conscience du fait qu'il faut envisager la totalité du cycle de vie des produits.

92. Le paragraphe 8 appelle les entreprises à prendre en compte les situations des consommateurs vulnérables et défavorisés lorsqu'elles commercialisent leurs produits. Ce terme de consommateurs défavorisés ou vulnérables se réfère à des consommateurs ou à des catégories de consommateurs spécifiques qui, en raison de caractéristiques ou de circonstances personnelles (telles que l'âge, la capacité mentale ou physique, l'éducation, le revenu, la langue ou l'éloignement), peuvent rencontrer des difficultés particulières sur les marchés actuels qui sont mondialisés et à forte intensité d'informations. Le paragraphe met par ailleurs en lumière l'importance croissante du commerce mobile et des autres formes de commerce électronique sur les marchés mondialisés. Les avantages que procure ce commerce sont importants et croissants. Les gouvernements ont consacré un temps considérable à examiner les moyens de faire en sorte que le niveau de transparence et d'efficacité de la protection des consommateurs ne soit pas moins élevé dans le cas du commerce électronique que dans celui des formes plus traditionnelles de commerce. 


\section{Science et technologie}

Les entreprises devraient :

1. S'efforcer de faire en sorte que leurs activités soient compatibles avec les politiques et plans scientifiques et technologiques des pays dans lesquels elles opèrent et, le cas échéant, contribuer au développement de la capacité d'innovation à l'échelon local et national.

2. Dans la mesure où cela est réalisable, adopter dans le cadre de leurs activités commerciales des pratiques permettant d'assurer le transfert et la diffusion rapide des technologies et du savoir-faire, en tenant dûment compte de la protection des droits de propriété intellectuelle.

3. Le cas échéant, mener des activités de développement scientifique et technologique dans les pays d'accueil de façon à répondre aux besoins du marché local, ainsi qu'employer du personnel du pays d'accueil dans des activités scientifiques et technologiques et encourager sa formation, compte tenu des besoins commerciaux.

4. Lorsqu'elles accordent des licences pour l'utilisation de droits de propriété intellectuelle ou qu'elles transfèrent des technologies par d'autres moyens, elles devraient le faire sur la base de conditions et modalités raisonnables et de manière à contribuer aux perspectives de développement durable à long terme du pays d'accueil.

5. Lorsque les objectifs commerciaux s'y prêtent, elles devraient établir localement des liens avec les universités et les établissements publics de recherche et participer à des projets de recherche en coopération avec les entreprises ou associations professionnelles locales.

\section{Commentaire sur la science et la technologie}

93. Dans une économie mondialisée et fondée sur le savoir, où les frontières nationales jouent un moindre rôle, même pour les entreprises de petite taille ou à vocation nationale, la possibilité d'accéder aux technologies et au savoir-faire et de les utiliser est essentielle pour améliorer les performances des entreprises. Cet accès est également important pour 
que se concrétisent les effets macro-économiques du progrès technologique, notamment la croissance de la productivité et la création d'emplois, dans le contexte du développement durable. Les entreprises multinationales sont le principal vecteur des transferts internationaux de technologie. Elles contribuent à la capacité nationale d'innovation de leur pays d'accueil dès lors qu'elles produisent de nouvelles technologies, en assurent la diffusion et en facilitent également l'utilisation dans les entreprises et institutions nationales. Les activités de R-D des entreprises multinationales, lorsqu'elles sont bien reliées au système national d'innovation, peuvent contribuer à accroître le progrès économique et social de leur pays d'accueil. Par ailleurs, la mise en place d'un système d'innovation dynamique dans le pays d'accueil élargit les débouchés commerciaux des entreprises multinationales.

94. Ce chapitre a donc pour but d'encourager les entreprises multinationales, en tenant compte des contraintes de faisabilité économique et de considérations de compétitivité ou autres, à diffuser dans les pays où elles opèrent les résultats des activités de R-D qu'elles mènent, en contribuant ainsi à la capacité d'innovation de leur pays d'accueil. Sur ce plan, les entreprises peuvent favoriser la diffusion des technologies en commercialisant des produits intégrant de nouvelles technologies, en accordant des licences pour des innovations de procédé, en embauchant et en formant du personnel spécialisé en science et technologie et en lançant des opérations de R-D en coopération. Lors de la vente ou de la concession de technologies, non seulement les modalités négociées devraient être raisonnables, mais il serait souhaitable que les entreprises multinationales envisagent les impacts à long terme des technologies sur le développement, l'environnement et d'autres domaines dans les pays d'origine et d'accueil. Dans leurs activités, les entreprises multinationales peuvent doter leurs filiales internationales et leurs soustraitants de capacités d'innovation et améliorer ces capacités. En outre, les entreprises multinationales peuvent attirer l'attention sur l'importance des infrastructures scientifiques et technologiques locales, aussi bien matérielles qu'institutionnelles. A cet égard, les entreprises multinationales peuvent contribuer utilement à la formulation, par les pays d'accueil, de cadres d'action propices au développement de systèmes d'innovation dynamiques. 


\section{Concurrence}

Les entreprises devraient :

1. Mener leurs activités d'une manière compatible avec tous les textes législatifs et réglementaires applicables, en prenant en compte le droit de la concurrence de tous les pays dans lesquels leurs activités risquent d'avoir des effets anticoncurrentiels.

2. S'abstenir de conclure ou d'exécuter des accords contraires à la concurrence, notamment des accords visant à :

a) fixer des prix ;

b) procéder à des soumissions concertées (ou collusions d'offres) ;

c) établir des limitations ou des quotas de production ; ou

d) se partager ou diviser les marchés en se répartissant des clients, des fournisseurs, des zones géographiques ou des lignes d'activités.

3. Dans le cadre d'enquêtes, coopérer avec les autorités de la concurrence, notamment, et sous réserve de la législation applicable et des précautions appropriées, en apportant des réponses aussi rapides et aussi exhaustives que possible aux demandes de renseignements, et en envisageant d'utiliser tous les instruments disponibles, par exemple les déclarations de renonciation au droit à la confidentialité, afin de favoriser une coopération efficace et efficiente entre les autorités chargées de mener les enquêtes.

4. Procéder régulièrement à des opérations de sensibilisation de leurs salariés à l'importance du respect de l'ensemble des lois et réglementations en matière de concurrence et, en particulier, former leurs dirigeants aux questions de concurrence.

\section{Commentaire sur la concurrence}

95. Ces recommandations soulignent l'importance, dans chaque pays, du droit et de la réglementation de la concurrence pour un fonctionnement efficace des marchés tant nationaux qu'internationaux, et réaffirment 
l'importance du respect de ces lois et de ces réglementations par les entreprises nationales et multinationales. Elles visent également à garantir que toutes les entreprises sont bien au fait des évolutions concernant la portée du droit de la concurrence, les recours et les sanctions prévus, ainsi que de l'importance de la coopération entre les autorités de la concurrence. L'expression «droit de la concurrence » est utilisée pour désigner les lois, y compris les lois antitrust et antimonopole qui, selon des modalités diverses, interdisent : a) les accords contraires à la concurrence; b) l'abus de pouvoir de marché ou de position dominante ; c) l'acquisition d'un pouvoir de marché ou d'une position dominante par des moyens autres qu'une performance efficiente ; ou encore d) l'amoindrissement substantiel de la concurrence ou l'exercice d'entraves significatives à une concurrence efficace par le biais de fusions ou d'acquisitions.

96. D'une manière générale, le droit et la politique de la concurrence interdisent: a) les ententes injustifiables; b) les autres accords contraires à la concurrence; c) les comportements anticoncurrentiels fondés sur l'exploitation ou l'élargissement d'une position dominante ou d'un pouvoir de marché ; et d) les fusions et acquisitions contraires à la concurrence. Aux termes de la Recommandation du Conseil de l'OCDE concernant une action efficace contre les ententes injustifiables (C(98)35/Final) adoptée en 1998, les accords anticoncurrentiels visés au point a) constituent des ententes injustifiables, mais la Recommandation tient compte des différences existant entre les différents droits nationaux des pays membres, notamment des différences dans les exceptions ou dispositions législatives prévoyant d'accorder une exception ou une autorisation pour des activités qui auraient été autrement interdites. Toutefois, cette recommandation ne signifie pas nécessairement que les entreprises ne devraient pas profiter de ces exceptions ou de ces dispositions. Les points b) et c) sont plus généraux parce que les effets d'autres formes d'accords et de comportements unilatéraux sont plus difficiles à apprécier, et parce que le consensus sur ce qu'il convient de considérer comme contraire à la concurrence est moins large.

97. La politique de la concurrence a pour objectif de contribuer au bien-être global et à la croissance économique générale en favorisant des conditions de marché dans lesquelles la nature, la qualité et le prix des biens et des services sont déterminés par le jeu des forces concurrentielles du marché. Outre qu'il bénéficie aux consommateurs et à l'économie d'un pays tout entière, un tel environnement concurrentiel est gratifiant pour les entreprises qui répondent efficacement à la demande des consommateurs. Bien souvent, les entreprises sont sources d'informations et de conseils lorsque les pouvoirs publics envisagent 
d'adopter des lois et des politiques risquant de nuire à l'efficience ou de réduire de toute autre manière la compétitivité des marchés.

98. Les entreprises doivent savoir que des lois sur la concurrence continuent d'être adoptées et qu'il est de plus en plus courant que ces lois interdisent les activités anticoncurrentielles menées à l'étranger si celles-ci devaient avoir des conséquences dommageables pour les consommateurs du pays concerné. Par ailleurs, du fait du caractère transnational des échanges et des investissements, un comportement anticoncurrentiel observé dans un pays est davantage susceptible d'avoir des conséquences dommageables dans un autre. En conséquence, un comportement qui est ou qui pourrait être légal dans un pays risque de plus en plus souvent d'être illégal dans un autre. Il faut donc que les entreprises prennent en compte aussi bien la réglementation du pays où elles opèrent que celle de tous les pays où leurs activités sont susceptibles de produire des effets.

99. Enfin, les entreprises devraient admettre que les autorités de la concurrence coopèrent de plus en plus étroitement pour enquêter sur les activités contraires à la concurrence et pour y faire échec. Pour des informations plus générales, on pourra se reporter à la Recommandation révisée du Conseil sur la coopération entre pays membres dans le domaine des pratiques anticoncurrentielles affectant les échanges internationaux [C(95)130/FINAL] ou encore à la Recommandation du Conseil sur le contrôle des fusions [C(2005)34]. Lorsque les autorités de la concurrence de différents pays examinent un même comportement, le fait, pour les entreprises, de faciliter la coopération entre les autorités concernées permettra de favoriser la cohérence et la pertinence des décisions et des recours et permettra également aux pouvoirs publics comme aux entreprises de réaliser des économies de coûts. 


\section{Fiscalité}

1. Il est important que les entreprises contribuent aux finances publiques des pays d'accueil en acquittant avec ponctualité les impôts dont elles sont redevables. En particulier, les entreprises devraient se conformer à la lettre comme à l'esprit des lois et règlements fiscaux des pays où elles opèrent. Respecter l'esprit de la loi signifie comprendre et suivre l'intention du législateur. Cette interprétation ne signifie pas qu'une entreprise doive acquitter un impôt supérieur au montant prévu par la loi. La discipline fiscale implique notamment de communiquer en temps voulu aux autorités compétentes les informations prescrites ou nécessaires à la détermination correcte des impôts dont sont passibles leurs activités et de se conformer dans leurs pratiques de prix de transfert au principe de pleine concurrence.

2. Les entreprises devraient considérer la gouvernance fiscale et la discipline fiscale comme des éléments importants de leurs mécanismes de contrôle et de leurs systèmes de gestion des risques au sens large. En particulier, les conseils d'administration devraient adopter des stratégies de gestion du risque fiscal qui permettent d'identifier et d'évaluer pleinement les risques financiers, réglementaires et de réputation associés à la fiscalité.

\section{Commentaire sur la fiscalité}

100. Une conduite responsable dans le domaine fiscal signifie que les entreprises devraient se conformer à la lettre comme à l'esprit des lois et règlements fiscaux de tous les pays dans lesquels elles exercent leurs activités, coopérer avec les autorités et leur communiquer les informations pertinentes ou requises par la loi. Une entreprise respecte l'esprit des lois et règlements fiscaux si elle prend des mesures raisonnables pour déterminer l'intention du législateur et interprète ces règles fiscales conformément à cette intention, à la lumière du texte de loi et de la jurisprudence contemporaine pertinente. Les transactions ne devraient pas être structurées en vue d'obtenir des résultats fiscaux sans rapport avec les conséquences économiques sous-jacentes de la 
transaction, sauf s'il existe une législation spécifique destinée à produire ce résultat. En pareil cas, l'entreprise devrait avoir de bonnes raisons de penser que la transaction est structurée de telle sorte qu'elle aboutit à un résultat fiscal qui n'est pas contraire à l'intention du législateur.

101. La discipline fiscale implique également de coopérer avec les autorités fiscales et de leur fournir les informations requises afin de garantir une application efficace et équitable des lois fiscales. Cette coopération suppose d'apporter des réponses rapides et complètes aux demandes de renseignements formulées par une autorité compétente conformément aux dispositions d'une convention fiscale ou d'un accord d'échange de renseignements. Cette exigence de communication d'informations connaît toutefois certaines limites. En particulier, les Principes directeurs établissent un lien entre les informations qui doivent être fournies et leur pertinence pour l'application des réglementations fiscales concernées. On prend ainsi en compte l'impératif d'équilibre entre la charge que représente pour les entreprises le respect des réglementations fiscales applicables et la nécessité, pour les autorités fiscales, de disposer d'informations complètes, actuelles et exactes pour pouvoir appliquer leurs réglementations fiscales.

102. Les engagements des entreprises en matière de coopération, de transparence et de discipline fiscale devraient se refléter dans des systèmes, structures et politiques de gestion des risques. Pour les entreprises constituées en société, les conseils d'administration sont en mesure de contrôler le risque fiscal par divers moyens. Par exemple, le conseil d'administration devrait, de manière anticipative, élaborer des principes appropriés de politique fiscale, et mettre en place des systèmes de contrôle interne garantissant que les actions des dirigeants sont conformes à l'analyse du risque fiscal effectuée par le conseil. Le conseil devrait être tenu informé de tout risque fiscal potentiellement grave, et les responsabilités devraient être établies pour l'exercice des fonctions internes de contrôle fiscal et de communication d'informations au conseil. Une stratégie globale de gestion des risques comportant un volet fiscal permettra à l'entreprise non seulement de faire preuve de civisme, mais aussi de gérer efficacement le risque fiscal, lui permettant ainsi de se prémunir contre d'importants risques financiers, réglementaires et de réputation.

103. Un membre d'un groupe multinational implanté dans un pays peut avoir d'étroites relations économiques avec d'autres membres du même groupe implantés dans d'autres pays et ces liens peuvent avoir une incidence sur les obligations fiscales de chacune des parties. En conséquence, les autorités fiscales peuvent avoir besoin d'informations 
en provenance de l'étranger pour se prononcer sur ces liens et déterminer l'impôt dû par le membre du groupe multinational sur leur territoire. Cette fois encore, les informations devant être fournies se limitent à ce qui est en rapport avec l'évaluation envisagée de ces liens économiques ou à ce qui est requis par la loi aux fins de cette évaluation en vue de déterminer correctement l'impôt dû par le membre du groupe multinational. Les entreprises multinationales devraient coopérer pour la communication de ces informations.

104. Une question importante du point de vue de la conduite responsable des entreprises dans le domaine de la fiscalité est celle des prix de transfert. L'expansion spectaculaire des échanges mondiaux et des investissements directs transnationaux (et le rôle de premier plan que jouent dans ces échanges et ces investissements les entreprises multinationales) signifie que les prix de transfert sont aujourd'hui un déterminant essentiel des obligations fiscales des membres d'un groupe multinational parce qu'ils conditionnent fortement la répartition de la base d'imposition entre les pays dans lesquels l'entreprise multinationale exerce ses activités. Le principe de pleine concurrence, qui figure à la fois dans le Modèle de convention fiscale de l'OCDE et le Modèle de convention des Nations unies concernant les doubles impositions entre pays développés et pays en développement, est la norme internationale utilisée pour répartir les bénéfices entre entreprises associées. L'application du principe de pleine concurrence évite le transfert inapproprié de bénéfices ou de pertes et minimise les risques de double imposition. Son application correcte nécessite que les entreprises multinationales coopèrent avec les autorités fiscales et leur communiquent toutes les informations pertinentes ou requises par la loi concernant le choix de la méthode de détermination des prix de transfert adoptée pour les transactions internationales entreprises par elles et par la partie associée. Il est admis qu'il est souvent difficile, aussi bien pour les entreprises multinationales que pour les administrations fiscales, de déterminer si les prix de transfert sont conformes à la norme (ou au principe) de pleine concurrence et que son application n'est pas une science exacte.

105. Le Comité des affaires fiscales de l'OCDE travaille en permanence à la mise au point de recommandations visant à assurer la conformité des prix de transfert au principe de pleine concurrence. Ses travaux ont abouti à la publication, en 1995, des Principes de 1'OCDE applicables en matière de prix de transfert à l'intention des entreprises multinationales et des administrations fiscales, qui ont donné lieu à la Recommandation du Conseil de l'OCDE sur la détermination des prix de transfert entre entreprises associées (les membres d'un groupe 
multinational constituent normalement des entreprises associées). Les Principes de l'OCDE applicables en matière de prix de transfert et cette Recommandation du Conseil sont régulièrement mis à jour afin de tenir compte des changements dans l'économie mondiale et de l'expérience des administrations fiscales et des contribuables confrontés aux prix de transfert. Le principe de pleine concurrence appliqué à l'attribution de bénéfices aux établissements stables afin de déterminer les droits d'imposition d'un pays d'accueil en vertu d'une convention fiscale a fait l'objet d'une Recommandation du Conseil de l'OCDE adoptée en 2008.

106. Les Principes de l'OCDE applicables en matière de prix de transfert concernent l'application du principe de pleine concurrence pour la détermination des prix de transfert des entreprises associées. Ils ont pour but d'aider les administrations fiscales (des pays membres de l'OCDE et des pays non membres) et les entreprises multinationales en indiquant des solutions mutuellement satisfaisantes aux problèmes que peuvent poser les prix de transfert, de façon à réduire les différends entre les administrations fiscales et, également, entre ces administrations et les entreprises multinationales, et à éviter ainsi de coûteux procès. Les entreprises multinationales sont encouragées à se conformer aux Principes de l'OCDE applicables en matière de prix de transfert, tels que modifiés et complétés, ${ }^{7}$ afin que leurs prix de transfert respectent le principe de pleine concurrence.

7. Un pays adhérent non membre de l'OCDE, le Brésil, n'applique pas les Principes de l'OCDE applicables en matière de prix de transfert sur le territoire qui relève de sa compétence. Par conséquent, l'utilisation des indications figurant dans ces Principes par les entreprises multinationales aux fins de déterminer le revenu imposable généré par leurs activités dans ce pays est sans objet à la lumière des obligations fiscales prévues par la législation de ce pays. Un autre pays non membre de l'OCDE, l'Argentine, souligne que l'application des Principes applicables en matière de prix de transfert n'est pas obligatoire dans les territoires relevant de sa compétence. 
Partie II

\section{Procédures de mise en ouvre des Principes directeurs de l'OCDE à l'intention des entreprises multinationales}





\section{Amendement de la décision du Conseil sur les principes directeurs de l'OCDE a l'intention des entreprises multinationales}

\section{LE CONSEIL,}

$\mathrm{Vu}$ la Convention relative à l'Organisation de coopération et de développement économiques, en date du 14 décembre 1960 ;

Vu la Déclaration de l'OCDE sur l'investissement international et les entreprises multinationales (la « Déclaration »), par laquelle les gouvernements des pays ayant adhéré (les «pays adhérents ») aux Principes directeurs à l'intention des entreprises multinationales (les «Principes directeurs ») recommandent conjointement aux entreprises multinationales opérant dans ou depuis leur territoire d'observer ces Principes directeurs ;

Reconnaissant que, dans la mesure où les opérations des entreprises multinationales s'étendent à travers le monde, la coopération internationale en matière de questions couvertes par la Déclaration devrait s'étendre à tous les pays ;

Vu le mandat du Comité de l'investissement, notamment en ce qui concerne les attributions du Comité à l'égard de la Déclaration [C(84)171(Final), renouvelé dans C/M(95)21] ;

Considérant le rapport sur le premier réexamen de la Déclaration de 1976 [C(79)102(Final)], le rapport sur le second réexamen de la Déclaration [C/MIN(84)5(Final)], le rapport sur le réexamen de 1991 de la Déclaration [DAFFE/IME(91)23] et le rapport sur le réexamen de 2000 des Principes directeurs [C(2000)96] ;

Vu la Seconde décision révisée du Conseil de juin 1984 [C(84)90], amendée en juin 1991 [C/MIN(91)7/ANN1] et abrogée le 27 juin 2000 [C(2000)96/FINAL] ; 
Considérant qu'il est souhaitable de renforcer les procédures permettant la tenue de consultations sur les questions couvertes par les Principes directeurs et d'encourager une application efficace de ces Principes ;

Sur proposition du Comité de l'investissement :

DÉCIDE :

\section{Points de contact nationaux}

1. Les pays adhérents créeront des Points de contact nationaux qui contribueront à renforcer l'efficacité des Principes directeurs en menant des activités de promotion, en répondant à des demandes de renseignements et en participant à la résolution des problèmes soulevés par la mise en œuvre des Principes directeurs dans des circonstances spécifiques, en prenant en compte les Lignes directrices de procédure cijointes. Les milieux d'affaires, les organisations représentant les travailleurs, d'autres organisations non gouvernementales et les autres parties intéressées seront informés de la mise en place de ces dispositifs.

2. Les Points de contact nationaux des différents pays coopéreront, en tant que de besoin, pour toute question de leur ressort couverte par les Principes directeurs. En règle générale, des discussions devront être entamées à l'échelon national avant que des contacts soient établis avec d'autres Points de contact nationaux.

3. Les Points de contact nationaux se réuniront régulièrement pour partager leurs expériences et faire rapport au Comité de l'investissement.

4. Les pays adhérents doteront leurs Points de contact nationaux de ressources humaines et financières de manière à ce qu'ils puissent s'acquitter efficacement de leurs responsabilités, en tenant compte de leurs priorités et pratiques budgétaires internes.

\section{Le Comité de l'investissement}

1. Le Comité de l'investissement («le Comité ») procédera, périodiquement ou à la demande d'un pays adhérent, à des échanges de vues sur des questions couvertes par les Principes directeurs et sur l'expérience tirée de leur application.

2. Le Comité invitera périodiquement le Comité consultatif économique et industriel auprès de l'OCDE (BIAC) et la Commission syndicale consultative auprès de l'OCDE (TUAC) («les organes consultatifs »), 
OECD Watch ainsi que d'autres partenaires internationaux, à exprimer leurs points de vue sur les questions visées par les Principes directeurs. A leur demande, des échanges de vues pourront aussi être organisés avec eux sur ces questions.

3. Le Comité s'engagera auprès des pays non adhérents sur les questions couvertes par les Principes directeurs de façon à promouvoir une conduite responsable des entreprises partout dans le monde, conformément aux dispositions des Principes directeurs, et à instaurer des règles du jeu équitables. Il s'efforcera également de coopérer avec les pays non adhérents ayant un intérêt spécifique pour les Principes directeurs afin d'en promouvoir auprès d'eux les principes et les normes.

4. Le Comité sera chargé d'apporter des éclaircissements sur les Principes directeurs. Les parties impliquées dans une circonstance spécifique ayant motivé une demande d'éclaircissement se verront offrir la possibilité d'exprimer leur point de vue, oralement ou par écrit. Le Comité ne tirera pas de conclusions sur la conduite d'entreprises déterminées.

5. Le Comité organisera des échanges de vues sur les activités menées par les Points de contact nationaux dans le but d'améliorer l'efficacité des Principes directeurs et de favoriser l'équivalence fonctionnelle des différents Points de contact nationaux.

6. En veillant au bon fonctionnement des Principes directeurs, conformément à ses attributions, le Comité tiendra dûment compte des Lignes directrices de procédure ci-jointes.

7. Le Comité fera un compte rendu périodique au Conseil sur les questions couvertes par les Principes directeurs. Dans ses comptes rendus, le Comité tiendra compte des rapports des Points de contact nationaux et des vues exprimées par les organes consultatifs, par OECD Watch, par d'autres partenaires internationaux et par des pays non adhérents le cas échéant.

8. Le Comité, en coopération avec les Points de contact nationaux, veillera, grâce à un "agenda proactif », à favoriser un respect effectif, par les entreprises, des principes et des normes énoncés dans les Principes directeurs. En particulier, il s'efforcera de rechercher des occasions de collaborer avec les organes consultatifs, avec OECD Watch, avec d'autres partenaires internationaux et d'autres parties prenantes pour encourager les contributions positives que les entreprises multinationales peuvent, en se référant aux Principes directeurs, apporter au progrès économique, environnemental et social afin 
d'instaurer un développement durable et pour aider les entreprises multinationales à identifier les risques d'incidences négatives associées à des produits, des régions, des secteurs ou des activités spécifiques, et à y répondre.

\section{Réexamen de la Décision}

La présente Décision sera réexaminée périodiquement. Le Comité soumettra des propositions à cet effet. 


\section{Lignes directrices de procédure}

\section{Points de contact nationaux}

Les Points de contact nationaux (PCN) ont pour mission de contribuer à une mise en œuvre efficace des Principes directeurs. Les PCN fonctionneront conformément à des critères essentiels (visibilité, accessibilité, transparence et responsabilité) afin de tendre vers l'objectif d'équivalence fonctionnelle.

\section{A. Modalités institutionnelles}

Conformément à l'objectif d'équivalence fonctionnelle et pour renforcer l'efficacité des Principes directeurs, les pays adhérents ont toute latitude pour organiser leur PCN, en recherchant le soutien actif des partenaires sociaux, notamment les milieux d'affaires et les organisations représentant les travailleurs, les autres organisations non gouvernementales et les autres parties intéressées.

En conséquence, les Points de contact nationaux :

1. Seront composés et organisés de façon à offrir une structure efficace pour le traitement des questions très diverses soulevées par les Principes directeurs et à pouvoir fonctionner en toute impartialité tout en respectant un niveau approprié de responsabilité vis-à-vis des pays adhérents.

2. Peuvent avoir recours à différentes formes d'organisation pour atteindre cet objectif. Un PCN peut être composé de représentants expérimentés d'un ou de plusieurs ministères, être un haut fonctionnaire ou un département administratif dirigé par un haut fonctionnaire, un groupe interministériel ou encore un groupe comprenant des experts indépendants. Des représentants des milieux d'affaires, des organisations représentant les travailleurs ou d'autres organisations non gouvernementales peuvent également en faire partie. 
3. Noueront et entretiendront des relations avec les représentants des milieux d'affaires, des organisations représentant les travailleurs et d'autres parties intéressées en mesure de contribuer au bon fonctionnement des Principes directeurs.

\section{B. Information et promotion}

Chaque Point de contact national devra :

1. Faire connaître les Principes directeurs et les diffuser par les moyens appropriés, y compris par des supports en ligne, et dans les langues nationales. Les investisseurs potentiels (désireux d'investir dans le pays ou à l'étranger) devront être informés en tant que de besoin sur les Principes directeurs.

2. Faire mieux connaître les Principes directeurs et leurs procédures de mise en œuvre, y compris en coopérant, le cas échéant, avec les milieux d'affaires, les organisations représentant les travailleurs, d'autres organisations non gouvernementales et le public intéressé.

3. Répondre aux demandes de renseignements sur les Principes directeurs émanant :

a) d'autres Points de contact nationaux ;

b) des milieux d'affaires, des organisations représentant les travailleurs, d'autres organisations non gouvernementales et du public ; et

c) des pouvoirs publics de pays non adhérents.

\section{Mise en ouvre dans des circonstances spécifiques}

Un Point de contact national contribuera à la résolution des questions soulevées par la mise en œuvre des Principes directeurs dans des circonstances spécifiques de manière impartiale, prévisible, équitable et compatible avec les principes et les normes énoncés dans les Principes directeurs. Il offrira une enceinte où ces questions pourront être examinées et aidera les milieux d'affaires, les organisations représentant les travailleurs, d'autres organisations non gouvernementales, et les autres parties intéressées à régler ces questions efficacement et promptement, et en conformité avec les lois applicables. Pour offrir cette assistance, le Point de contact national :

1. Procédera à une première évaluation de l'intérêt des questions soulevées pour déterminer si elles méritent d'être approfondies et répondra aux parties impliquées. 
2. Si les questions posées justifient un examen approfondi, proposera de bons offices pour aider les parties impliquées à les régler. À cette fin, le PCN consultera ces parties et, selon les cas :

a) sollicitera l'avis d'autorités compétentes et/ou de représentants des milieux d'affaires, des organisations représentant les travailleurs, d'autres organisations non gouvernementales et d'experts ;

b) consultera le PCN de l'autre pays concerné ou des autres pays concernés ;

c) sollicitera l'avis du Comité de l'investissement s'il a des doutes sur l'interprétation des Principes directeurs dans le cas d'espèce ;

d) proposera et, avec l'accord des parties impliquées, facilitera l'accès à des moyens consensuels et non contentieux, tels que la conciliation ou la médiation, afin d'aider les parties à résoudre les problèmes.

3. A l'issue des procédures et après consultation des parties impliquées, rendra publics les résultats des procédures, en tenant compte de la nécessité de protéger les informations sensibles, qu'il s'agisse d'informations commerciales ou d'autres informations relatives aux parties prenantes, en publiant :

a) un communiqué dans le cas où il décide que les questions soulevées ne justifient pas un examen plus approfondi. Dans ce communiqué, le PCN doit au minimum présenter les questions soulevées et donner les motifs de sa décision ;

b) un rapport dans le cas où les parties sont parvenues à un accord sur les questions soulevées. Dans ce rapport, le PCN doit au minimum présenter les questions soulevées, les procédures qu'il a engagées pour aider les parties et indiquer à quel moment un accord a été conclu. Les informations relatives à la teneur de l'accord n'y figureront que si les parties concernées donnent leur approbation ;

c) un communiqué dans le cas où aucun accord n'a été conclu ou lorsqu'une des parties ne souhaite pas participer à la procédure. Dans ce communiqué, le PCN doit au minimum présenter les questions soulevées, les raisons pour lesquelles il a décidé qu'elles justifiaient un examen approfondi et les procédures qu'il a engagées pour aider les parties. Le PCN formulera des recommandations appropriées sur la mise en œuvre des Principes directeurs, qui devront figurer dans le communiqué. Le cas échéant, il pourra 
également indiquer les raisons pour lesquelles il n'a pas été possible de parvenir à un accord.

Le PCN fera rapidement connaître au Comité les résultats des procédures qu'il aura engagées au titre de circonstances spécifiques.

4. Afin de faciliter le règlement des questions soulevées, prendra les mesures appropriées en vue de protéger les informations sensibles, commerciales ou autres, ainsi que les intérêts des autres parties prenantes impliquées dans les circonstances spécifiques. Pendant la durée des procédures prévues au paragraphe 2, la confidentialité des travaux sera assurée. À l'issue des procédures, si les parties impliquées ne sont pas tombées d'accord sur une résolution des questions soulevées, elles seront libres de s'exprimer et de discuter de ces questions. En revanche, les informations et les avis avancés durant les travaux par une autre partie impliquée resteront confidentiels, à moins que cette partie n'accepte qu'ils soient divulgués ou que ce soit contraire aux dispositions de la législation nationale.

5. Si des questions se posent dans des pays n'ayant pas adhéré aux Principes directeurs, prendra des mesures afin de parvenir à une meilleure compréhension des questions soulevées, et suivra la présente procédure en tant que de besoin et dans la mesure du possible.

\section{D. Établissement de rapports}

1. Chaque PCN fera chaque année un rapport au Comité.

2. Ce rapport devra contenir des informations sur la nature et le résultat des activités menées par le PCN, y compris les activités de mise en œuvre dans des circonstances spécifiques.

\section{Comité de l'investissement}

1. Le Comité étudiera les demandes d'assistance des PCN dans le cadre de leurs activités, y compris en cas de doute quant à l'interprétation des Principes directeurs dans des circonstances particulières.

2. Dans le but d'améliorer l'efficacité des Principes directeurs et de favoriser l'équivalence fonctionnelle des PCN, le Comité :

a) étudiera les rapports des PCN ;

b) étudiera les demandes dûment motivées émanant d'un pays adhérent, d'un organe consultatif ou d'OECD Watch sur le point de 
savoir si un PCN s'acquitte ou non de sa mission en ce qui concerne le règlement de questions soulevées dans des circonstances spécifiques ;

c) envisagera d'apporter une clarification dans les cas où un pays adhérent, un organe consultatif ou OECD Watch formulerait une demande dûment motivée concernant la pertinence de l'interprétation des Principes directeurs donnée par un PCN dans des circonstances spécifiques ;

d) formulera des recommandations, si nécessaire, pour améliorer le fonctionnement des PCN et rendre plus efficace la mise en œuvre des Principes directeurs ;

e) coopérera avec des partenaires internationaux ;

f) s'engagera auprès des pays non adhérents intéressés sur les questions couvertes par les Principes directeurs et leur mise en œuvre.

3. Le Comité pourra solliciter et examiner l'avis d'experts sur toutes les questions couvertes par les Principes directeurs. Il décidera des procédures à suivre à cette fin.

4. Le Comité exercera ses attributions efficacement et promptement.

5. Dans l'exercice de sa mission, le Comité sera assisté par le Secrétariat de l'OCDE qui, selon les orientations générales données par le Comité de l'investissement et sous réserve du Programme de travail et budget de l'Organisation :

a) servira de source d'information de référence pour les PCN ayant des questions sur la promotion et la mise en œuvre des Principes directeurs ;

b) recueillera et rendra publiques les informations appropriées relatives aux tendances récentes et aux pratiques se faisant jour à propos des activités de promotion des PCN et de la mise en œuvre des Principes directeurs dans des circonstances spécifiques. Le Secrétariat élaborera des formats-types unifiés pour la rédaction des rapports, ce qui lui permettra de constituer et de maintenir une base de données à jour sur les circonstances spécifiques et de procéder régulièrement à des analyses de ces circonstances spécifiques ;

c) facilitera les activités d'apprentissage mutuel, notamment les évaluations mutuelles volontaires, ainsi que les activités de renforcement des capacités et de formation, en particulier à 
l'intention des PCN de pays nouvellement adhérents, sur les procédures de mise en œuvre des Principes directeurs telles que les mesures visant à encourager et à faciliter la conciliation et la médiation ;

d) facilitera la coopération entre les PCN en tant que de besoin; et

e) fera la promotion des Principes directeurs dans les enceintes et réunions internationales appropriées et apportera son aide aux PCN et au Comité dans leurs efforts visant à faire mieux connaître les Principes directeurs aux pays non adhérents. 


\section{Commentaire sur les procédures de mise en œuvre des Principes directeurs de l'OCDE à l'intention des entreprises multinationales}

1. La Décision du Conseil concrétise l'engagement des pays adhérents de participer à la mise en œuvre des recommandations contenues dans le texte des Principes directeurs. Des Lignes directrices de procédure concernant les Points de contact nationaux et le Comité de l'investissement sont annexées à cette Décision du Conseil.

2. La Décision du Conseil souligne les responsabilités fondamentales des pays adhérents en ce qui concerne les PCN. Ces responsabilités fondamentales peuvent être résumées comme suit :

- Instituer des PCN (qui tiendront compte des lignes directrices de procédure annexées à la Décision) et informer les parties intéressées de l'existence d'un dispositif ayant trait aux Principes directeurs.

- Mettre à leur disposition des ressources humaines et financières nécessaires.

- Permettre aux PCN de différents pays de coopérer mutuellement en tant que de besoin.

- Permettre aux PCN de se réunir régulièrement et de faire des rapports au Comité.

3. La Décision du Conseil définit également les responsabilités du Comité de l'investissement concernant les Principes directeurs :

- Organiser des échanges de vues sur les questions se rapportant aux Principes directeurs.

- Apporter des éclaircissements en tant que de besoin.

- Organiser des échanges de vues sur les activités des PCN.

- Faire rapport au Conseil de l'OCDE sur les Principes directeurs. 
4. Le Comité de l'investissement est l'organe de l'OCDE chargé de superviser l'application des Principes directeurs. Cette responsabilité ne s'applique pas uniquement aux Principes directeurs eux-mêmes, mais à tous les éléments de la Déclaration (instruments relatifs au traitement national, aux stimulants et obstacles à l'investissement international ainsi qu'aux obligations contradictoires). Le Comité cherche à s'assurer que chaque élément de la Déclaration est bien compris et respecté, et que tous ces éléments sont complémentaires et s'articulent harmonieusement.

5. Témoignant de l'intérêt croissant que présente la conduite responsable des entreprises pour les pays situés en dehors de la zone OCDE, la Décision préconise un engagement et une coopération avec les pays non adhérents sur les questions couvertes par les Principes directeurs. Cette disposition permet au Comité d'organiser des réunions spéciales avec des pays non adhérents intéressés afin de faire mieux connaître les principes et les normes énoncés dans les Principes directeurs ainsi que les procédures permettant leur mise en œuvre. Sous réserve du respect des procédures de l'OCDE applicables en la matière, le Comité peut aussi associer ces pays à des activités ou projets spécifiques portant sur la conduite responsable des entreprises, notamment en les invitant à ses réunions ou aux Tables rondes sur la responsabilité des entreprises.

6. Dans le cadre de son «agenda proactif », le Comité coopérera avec les PCN et recherchera toutes les occasions de collaborer avec les organes consultatifs, avec OECD Watch et avec d'autres partenaires internationaux. On trouvera au paragraphe 18, à l'intention des PCN, d'autres orientations sur cette question.

\section{Commentaire sur les lignes directrices de procédure pour les Points de contact nationaux}

7. Les Points de contact nationaux ont un rôle important à jouer afin d'améliorer la notoriété et l'efficacité des Principes directeurs. Certes, il incombe aux entreprises de respecter les Principes directeurs dans leur comportement quotidien, mais les pouvoirs publics peuvent contribuer à améliorer l'efficacité des procédures conçues pour leur mise en œuvre. À cette fin, les gouvernements considèrent que des orientations plus précises concernant les modalités d'action et les activités des PCN sont nécessaires, y compris sous la forme de réunions régulières et d'une supervision de la part du Comité de l'investissement. 
8. La majorité des fonctions décrites dans les Lignes directrices de procédure de la Décision ne sont pas nouvelles, mais reflètent l'expérience acquise et les recommandations formulées au fil du temps. Parce qu'elles sont plus explicites, le fonctionnement envisagé des mécanismes de mise en œuvre des Principes directeurs gagne en transparence. Toutes les fonctions sont désormais définies en quatre points dans les Lignes directrices de procédure relatives aux PCN : modalités institutionnelles, information et promotion, mise en œuvre dans des circonstances spécifiques et établissement de rapports.

9. Ces quatre points sont précédés d'un paragraphe introductif qui définit la finalité principale des PCN ainsi que les critères essentiels à la promotion de la notion d' "équivalence fonctionnelle». Dans la mesure où les pouvoirs publics ont une certaine latitude dans l'organisation de leur PCN, ceux-ci se doivent de fonctionner de manière visible, accessible, transparente et responsable. Ces critères guideront les PCN dans l'exercice de leurs fonctions et seront également utiles au Comité lorsqu'il discutera de la façon dont les PCN s'acquittent de leur mission.

\section{Critères essentiels d'équivalence fonctionnelle concernant les activités des PCN}

Visibilité. En vertu de la Décision, les gouvernements adhérents s'engagent à désigner des PCN et à informer les milieux d'affaires, les organisations représentant les travailleurs et les autres parties intéressées, y compris les ONG, de la mise en place des dispositifs associés aux PCN pour la mise en œuvre des Principes directeurs. Les gouvernements sont censés publier des informations sur leur PCN et jouer un rôle actif dans la promotion des Principes directeurs, ce qui pourra les amener à organiser des séminaires et des réunions au sujet de cet instrument. Ces réunions pourront être organisées en coopération avec les entreprises, les organisations syndicales, les ONG et d'autres parties intéressées, mais pas nécessairement avec tous ces groupes à chaque occasion.

Accessibilité. La facilité d'accès aux PCN est importante pour leur bon fonctionnement. Ceci signifie que les entreprises, les syndicats, les ONG et le public en général doivent pouvoir y recourir facilement. Les moyens de communication électroniques peuvent faciliter les choses à cet égard. Les PCN devront répondre efficacement et promptement à toutes les demandes légitimes d'informations et traiter les questions spécifiques soulevées par les parties concernées. 
Transparence. La transparence est un critère important en ce qu'elle concourt à la responsabilité du PCN et à la confiance du public. Ainsi, il est posé comme principe général que les activités des PCN doivent être transparentes. Néanmoins, lorsqu'un PCN proposera ses « bons offices » pour la mise en œuvre des Principes directeurs dans des circonstances spécifiques, il faudra, à des fins d'efficacité, prendre les mesures nécessaires pour garantir la confidentialité des travaux. La transparence s'appliquera aux résultats, sauf si une mise en œuvre efficace des Principes directeurs impose le respect de la confidentialité.

Responsabilité. Le fait, pour les PCN, de contribuer plus activement à mieux faire connaître les Principes directeurs - et d'être à même de faciliter le règlement de questions délicates entre les entreprises et les collectivités au sein desquelles elles opèrent - attirera également l'attention du public sur leurs activités. Au niveau national, les parlements pourraient avoir un rôle à jouer. Les rapports annuels et les réunions régulières des PCN seront l'occasion de partager l'expérience acquise et d'encourager l'adoption de « pratiques exemplaires » par les PCN. Le Comité organisera des échanges de vues, de manière à partager l'expérience acquise et à évaluer l'efficacité des activités des PCN.

\section{Modalités institutionnelles}

10. La personne ou l'entité placée à la tête du PCN devrait être à même de susciter la confiance des partenaires sociaux et des autres parties prenantes, et de promouvoir les Principes directeurs auprès du public.

11. Quelle que soit la structure retenue par les pouvoirs publics pour leur $\mathrm{PCN}$, ils peuvent par ailleurs mettre sur pied des organes consultatifs ou des organes de contrôle réunissant plusieurs parties prenantes afin d'aider les PCN à remplir leur mission.

12. Les PCN, quelle que soit leur composition, doivent instaurer et développer des relations avec les représentants des milieux d'affaires, des organisations représentant les travailleurs, d'autres organisations non gouvernementales et d'autres parties intéressées. 


\section{Information et promotion}

13. Les fonctions des $\mathrm{PCN}$ se rattachant à l'information et à la promotion sont fondamentales pour accroître la notoriété des Principes directeurs.

14. Les PCN doivent mieux faire connaitre les Principes directeurs et les diffuser en ligne ainsi que par d'autres moyens appropriés, y compris dans les langues nationales. Il sera possible de se procurer les versions du texte en anglais et en français auprès de l'OCDE et il est recommandé que des liens avec le site Internet des Principes directeurs soient mis en place. Le cas échéant, les PCN donneront aux investisseurs potentiels désireux d'investir dans le pays ou à l'étranger des informations sur les Principes directeurs.

15. Les PCN doivent donner des informations sur les procédures que les parties doivent suivre lorsqu'elles soulèvent une question ou bien sont sollicitées dans le cadre d'une circonstance spécifique. Ils doivent en particulier dispenser des conseils concernant les informations nécessaires pour invoquer une circonstance spécifique, les obligations qui incombent aux parties impliquées dans une circonstance spécifique, notamment en matière de confidentialité, et donner des renseignements sur les procédures qu'ils suivront et des indications sur le calendrier de la mise en œuvre de ces procédures.

16. Dans leur action de promotion des Principes directeurs, les PCN coopéreront avec un large éventail d'organisations et de personnes, y compris selon les circonstances, les milieux d'affaires, les organisations représentant les travailleurs, d'autres organisations non gouvernementales et d'autres parties intéressées. Ces organisations ont un grand rôle à jouer pour faire mieux connaître les Principes directeurs et leurs réseaux institutionnels offrent des possibilités de promotion qui, si elles sont bien utilisées à cette fin, permettront d'amplifier largement les efforts déployés en ce sens par les PCN.

17. Répondre aux demandes légitimes de renseignements figure au nombre des missions fondamentales des PCN. Trois groupes sont particulièrement concernés : i) les autres PCN (la Décision comporte une disposition spécifique à cet effet) ; ii) les milieux d'affaires, les organisations représentant les travailleurs, les autres organisations non gouvernementales et le public ; iii) les pouvoirs publics des pays n'ayant pas adhéré aux Principes directeurs. 


\section{«Agenda proactif»}

18. Conformément à «l'agenda proactif» du Comité de l'investissement, les PCN doivent rester en contact régulier, notamment par le biais de réunions, avec les partenaires sociaux et les autres parties prenantes de façon à :

a) examiner les évolutions nouvelles et les pratiques se faisant jour en matière de conduite responsable des entreprises ;

b) favoriser la contribution positive que les entreprises peuvent apporter au progrès économique, social et environnemental ;

c) participer le cas échéant aux initiatives collectives visant à identifier les risques d'incidences négatives liées à des produits, des régions, des secteurs ou des activités spécifiques, et à répondre à ces risques.

\section{Apprentissage mutuel}

19. Outre qu'ils doivent contribuer aux travaux d'amélioration de l'efficacité des Principes directeurs menés par le Comité, les PCN devront prendre part à des activités conjointes d'apprentissage mutuel. En particulier, ils sont encouragés à participer à des évaluations horizontales, des examens mutuels thématiques ou encore des évaluations mutuelles volontaires des PCN. Cet apprentissage mutuel peut se dérouler dans le cadre de réunions organisées à l'OCDE, ou passer par une coopération directe entre les PCN.

\section{Mise en auvre dans des circonstances spécifiques}

20. Lorsque des questions se posent concernant la mise en œuvre des Principes directeurs dans des circonstances spécifiques, le PCN devra aider à les résoudre. Cette section des Lignes directrices de procédure contient des orientations à l'intention des $\mathrm{PCN}$ afin de les aider à gérer ces circonstances spécifiques.

21. L'efficacité de la procédure des circonstances spécifiques dépend de la bonne foi de toutes les parties concernées. Dans ce contexte, agir de «bonne foi » signifie répondre promptement, respecter le cas échéant la confidentialité des informations, s'abstenir de déformer les faits, de menacer de représailles les parties concernées par la procédure ou d'exercer effectivement de telles représailles et s'engager sincèrement 
dans les procédures avec la volonté de parvenir à une résolution des questions soulevées en référence aux Principes directeurs.

\section{Principes généraux applicables aux circonstances spécifiques}

22. Conformément aux critères fondamentaux d'équivalence fonctionnelle de leurs activités, les PCN devront traiter les circonstances spécifiques en respectant quatre principes :

Impartialité: Les PCN devront résoudre les affaires invoquant des circonstances spécifiques en toute impartialité.

Prévisibilité : Les PCN devront veiller à la prévisibilité de leurs décisions en fournissant au public des informations claires et facilement accessibles sur le rôle qu'ils jouent dans la résolution des affaires relevant de circonstances spécifiques, notamment la nature de leurs bons offices, les différentes étapes de la procédure propre aux circonstances spécifiques avec un calendrier indicatif, ou encore le rôle qu'ils peuvent être amenés à jouer pour contrôler la mise en application des accords conclus entre les parties.

Équité : Les PCN devront s'assurer que les parties sont à même de s'engager dans une procédure dans des conditions justes et équitables, par exemple en permettant, dans des conditions raisonnables, l'accès à des sources de renseignements utiles pour la procédure.

Conformité aux Principes directeurs : Les PCN doivent fonctionner en conformité avec les principes et les normes énoncés dans les Principes directeurs.

\section{Coordination entre les PCN dans des circonstances spécifiques}

23. Généralement, les questions seront traitées par le PCN du pays dans lequel elles auront été soulevées. S'il s'agit de pays adhérents, ces questions seront d'abord examinées à l'échelon national et ensuite, le cas échéant, abordées dans un cadre bilatéral. Dans le cadre de ses efforts visant à aider les parties en présence à résoudre la question, le PCN du pays d'accueil devra consulter le PCN du pays d'origine. Ce dernier devra s'efforcer de fournir promptement l'aide appropriée qui lui aura été demandée par le PCN du pays d'accueil.

24. Lorsque des questions concernent des activités exercées par une entreprise dans plusieurs pays adhérents, ou des activités exercées par 
un groupe d'entreprises organisées en consortium, en co-entreprise ou autre structure similaire et installées dans différents pays adhérents, les PCN concernés devront se consulter afin de se mettre d'accord sur le PCN qui sera au premier chef chargé d'aider les parties. Les PCN peuvent demander l'aide du Président du Comité de l'investissement pour parvenir à un tel accord. Le PCN principal devra consulter les autres PCN qui devront lui fournir à sa demande l'assistance appropriée. Faute d'accord entre les parties, le PCN principal devra prendre une décision finale en concertation avec les autres PCN.

\section{Évaluation initiale}

25. Lors de l'évaluation initiale de l'opportunité d'examiner la question de manière plus approfondie, le PCN devra déterminer si la question soulevée l'est de bonne foi et est en rapport avec les Principes directeurs. Dans ce contexte, le PCN tiendra compte :

- De l'identité de la partie concernée et de son intérêt dans l'affaire ;

- Du caractère significatif de la question et des éléments fournis à l'appui ;

- Du lien apparent entre les activités de l'entreprise et la question soulevée dans la circonstance spécifique ;

- De la pertinence des lois et procédures applicables, notamment des décisions de justice ;

- De la manière dont des questions similaires sont (ou ont été) traitées au niveau national ou international ;

- De l'intérêt que présente l'examen de la question concernée au regard des objectifs visés par les Principes directeurs et de l'efficacité de leur mise en œuvre.

26. Lorsqu'ils évaluent l'importance, pour une procédure relative à une circonstance spécifique, d'autres actions nationales ou internationales traitant parallèlement de questions comparables, les PCN ne doivent pas décider que les questions ne justifient pas un examen plus approfondi simplement parce que des actions parallèles ont existé, sont en cours ou pourraient être menées par les parties concernées. Les PCN devront essayer de déterminer si, en proposant leurs bons offices, ils pourraient contribuer de manière positive à la résolution des questions soulevées et si cela ne risque pas d'entraîner un préjudice grave pour l'une ou l'autre des parties engagées dans ces autres procédures, ou de constituer une atteinte à l'autorité de la justice. Pour ce faire, les PCN devront tenir 
compte des pratiques en vigueur dans les autres PCN et, le cas échéant, consulter les instances devant lesquelles ces actions parallèles sont menées ou pourraient l'être. Les parties devront également aider les PCN à examiner ces questions en leur fournissant toutes les informations utiles sur les actions parallèles.

27. Après son évaluation initiale, le $\mathrm{PCN}$ devra donner sa réponse aux parties concernées. S'il décide que la question ne mérite pas d'être approfondie, il informera les parties des motifs de sa décision.

\section{Assistance aux parties}

28. Si les questions soulevées justifient un examen plus approfondi, le PCN en discutera avec les parties impliquées et proposera ses «bons offices » pour tenter de contribuer de manière informelle à leur règlement. Le cas échéant, les PCN suivront la procédure prévue aux paragraphes $\mathrm{C} 2$ a) à C $2 \mathrm{~d}$ ). Ils pourront demander l'avis des autorités compétentes, ainsi que des représentants des milieux d'affaires, des organisations syndicales, d'autres organisations non gouvernementales et d'experts. Des consultations avec les PCN d'autres pays ou des demandes d'assistance sur des points touchant à l'interprétation des Principes directeurs pourront également contribuer à la résolution des questions soulevées.

29. Dans le cadre de leur offre de bons offices, et si les questions soulevées l'exigent, les PCN offriront ou faciliteront l'accès à des procédures consensuelles, non contentieuses, telles que la conciliation ou la médiation, afin d'aider au règlement des questions soulevées. Comme c'est le cas pour les pratiques admises de conciliation et de médiation, ces procédures ne seront utilisées qu'avec l'accord des parties concernées et à condition qu'elles s'engagent à agir de bonne foi pendant la procédure.

30. En proposant leurs bons offices, les PCN pourront prendre des mesures destinées à protéger l'identité des parties impliquées lorsqu'il y aura de solides raisons de croire que la divulgation de ces informations risquerait de nuire à l'une ou plusieurs des parties. Cela concerne en particulier les circonstances où il pourrait être nécessaire de dissimuler à l'entreprise impliquée l'identité d'une ou de plusieurs parties. 


\section{Conclusion des procédures}

31. Les PCN devront toujours rendre publiques les conclusions d'une procédure de circonstance spécifique, conformément aux paragraphes $\mathrm{C}$ 3 et C-4 des Lignes directrices de procédure.

32. Si après avoir procédé à son évaluation initiale, un $\mathrm{PCN}$ décide que les questions soulevées dans le cadre de la circonstance spécifique ne méritent pas d'être approfondies, il devra en faire état dans un communiqué public après avoir consulté les parties concernées et en tenant compte de la nécessité de respecter la confidentialité des informations commerciales ou autres sensibles. Si, à partir des conclusions de son évaluation initiale, un PCN estime qu'il serait contraire à l'équité de dévoiler publiquement l'identité d'une partie dans un communiqué relatif à sa décision, il pourra rédiger ce communiqué de façon à protéger l'identité de la partie concernée.

33. Un PCN qui décide que des questions méritent un examen approfondi et offre ses bons offices aux parties concernées peut aussi en faire état publiquement dans un communiqué.

34. Si les parties concernées parviennent à un accord sur les questions soulevées, elles doivent indiquer dans celui-ci selon quelles modalités et dans quelle mesure la teneur de leur accord sera rendue publique. Le $\mathrm{PCN}$, en concertation avec les autres parties, publiera un rapport présentant les résultats de la procédure. Les parties peuvent également convenir de demander l'aide du PCN pour assurer le suivi de la mise en œuvre de leur accord et le PCN pourra exercer ce suivi selon les modalités convenues entre les parties et lui.

35. Si les parties impliquées ne parviennent pas à s'entendre sur les questions soulevées, ou si le PCN estime que l'une ou plusieurs des parties à la procédure de circonstance spécifique refuse de s'engager ou d'y participer de bonne foi, il publiera un communiqué et formulera le cas échéant des recommandations sur la mise en œuvre des Principes directeurs. Cette procédure établit de manière claire que le $\mathrm{PCN}$ doit publier un communiqué, même s'il estime qu'une recommandation spécifique n'est pas nécessaire. Ce communiqué doit mentionner l'identité des parties concernées, les questions soulevées, la date à laquelle ces questions ont été portées à l'attention du PCN, les recommandations éventuelles du PCN et toute observation qu'il jugera utile d'inclure sur les raisons pour lesquelles il n'a pas été possible de parvenir à un accord. 
36. Le PCN devra rédiger un projet de communiqué et donner aux parties la possibilité de le commenter. Toutefois, ce communiqué est celui du PCN et il lui appartiendra, à sa discrétion, de décider ou non de modifier le projet de communiqué en fonction des commentaires des parties. Si le PCN formule des recommandations à l'intention des parties, il pourra être opportun, dans certains cas, qu'il vérifie ensuite auprès des parties comment elles ont réagi à ces recommandations. Si le PCN juge un tel suivi approprié, le calendrier de la procédure devra être indiqué dans son communiqué.

37. Les communiqués et les rapports sur les résultats des procédures publiés par les PCN peuvent être utiles aux pouvoir publics pour l'administration de leurs programmes et de leurs politiques. Afin de favoriser la cohérence des politiques, les PCN sont ainsi encouragés à transmettre leurs communiqués et leurs rapports à des organismes publics lorsqu'ils savent que la teneur de ces documents peut intéresser un organisme spécifique dans la conduite de ses politiques ou de ses programmes. Cette disposition ne modifie en rien le caractère volontaire des Principes directeurs.

\section{Transparence et confidentialité}

38. La transparence est reconnue comme un principe général d'action des PCN dans leurs rapports avec le public (voir ci-avant le paragraphe 9 sur les « critères essentiels »). Toutefois, le paragraphe $\mathrm{C} 4$ des Lignes directrices de procédure admet qu'il existe des circonstances particulières où la confidentialité est importante. Le PCN prendra les mesures nécessaires afin de protéger les données sensibles des entreprises. De même, d'autres données telles que l'identité des personnes impliquées dans la procédure devront rester confidentielles à des fins de plus grande efficacité des Principes directeurs. Il est entendu que les faits et arguments avancés par les parties sont inclus dans la notion de «travaux ». Néanmoins, il est essentiel de trouver un équilibre entre transparence et confidentialité si l'on veut que les procédures associées aux Principes directeurs suscitent la confiance et qu'elles soient mises en œuvre de manière efficace. De ce fait, si le paragraphe C 4 précise que les travaux associés à la mise en œuvre resteront normalement confidentiels, leur résultat sera normalement transparent. 


\section{Questions soulevées dans des pays non adhérents}

39. Comme l'indique l'article 2 du chapitre sur les « Concepts et principes ", les entreprises sont encouragées à respecter les Principes directeurs partout où elles exercent leurs activités, en tenant compte de la situation particulière de chaque pays d'accueil.

- Si des questions se rapportant aux Principes directeurs se posent dans des pays non adhérents, les PCN des pays d'origine prendront des mesures afin de parvenir à une meilleure compréhension des questions soulevées. S'il peut se révéler parfois impraticable d'accéder à certaines informations utiles, ou de réunir toutes les parties impliquées, le $\mathrm{PCN}$ peut être néanmoins à même de procéder à des investigations. Entrer en contact avec la direction de l'entreprise du pays d'accueil et, éventuellement, avec les ambassades et les autorités compétentes du pays non adhérent, peut faire partie de ces mesures.

- Des contradictions avec les lois, les règlements et les politiques des pays hôtes peuvent rendre la mise en ouvre effective des Principes directeurs dans des circonstances spécifiques plus difficile dans des pays non adhérents que dans les pays adhérents. Comme noté dans le commentaire du chapitre sur les Principes généraux, si les Principes directeurs vont au-delà des législations dans de nombreux cas, ils ne doivent pas et n'ont pas pour objet de mettre l'entreprise dans une situation où elle devrait faire face à des obligations contradictoires.

- Les parties impliquées devront être avisées des limitations inhérentes à la mise en œuvre des Principes directeurs dans les pays non adhérents.

- Les problèmes posés par l'application des Principes directeurs dans les pays non adhérents pourraient également être examinés lors des réunions des PCN afin de contribuer à accroître l'expérience relative au traitement des questions soulevées dans ces pays.

\section{Calendrier indicatif}

40. Une procédure engagée dans le cadre d'une circonstance spécifique se déroule en trois étapes :

1. Évaluation initiale et décision sur l'opportunité de proposer ses bons offices pour aider les parties: Les PCN devront s'efforcer de conclure leur évaluation initiale dans un délai de trois mois, bien qu'un délai supplémentaire puisse quelquefois être nécessaire pour recueillir les informations indispensables à une décision éclairée. 
2. Assistance aux parties s'efforçant de résoudre les questions soulevées: Si un PCN décide de proposer ses bons offices, il devra s'efforcer de faciliter promptement la résolution des questions soulevées. Reconnaissant que les progrès permis par ces bons offices, y compris au moyen de procédures de médiation et de consultation, dépendent en dernier ressort des parties concernées, le PCN devra, après consultation de ces parties, définir un calendrier raisonnable de discussion entre elles pour résoudre les questions soulevées. Si elles n'arrivent pas à conclure un accord dans le délai ainsi imparti, le PCN devra consulter les parties pour déterminer si cela vaut la peine qu'il continue à leur apporter son aide ; s'il conclut que la poursuite de la procédure ne risque pas d'aboutir à un résultat, il doit mettre un terme à la procédure et préparer un communiqué.

3. Conclusion des procédures : Le PCN devra publier son communiqué ou son rapport dans les trois mois suivant la conclusion de la procédure.

41. En règle générale, les $\mathrm{PCN}$ devront s'efforcer de conclure les procédures dans les douze mois suivant la réception de la requête relative à une circonstance spécifique, étant toutefois entendu que ce délai pourra être étendu si les circonstances l'imposent, par exemple si la question est soulevée dans un pays n'ayant pas adhéré aux Principes directeurs.

\section{Rapports au Comité de l'investissement}

42. L'établissement de rapports sera une responsabilité importante des PCN qui permettra également de constituer une base de connaissances et d'acquérir un savoir-faire pour améliorer l'efficacité des Principes directeurs. Sachant cela, les PCN remettront au Comité de l'investissement des rapports qui permettront d'inclure dans le Rapport annuel sur les Principes directeurs de l'OCDE des informations relatives à toutes les circonstances spécifiques invoquées par des parties, à la fois celles qui sont encore au stade de l'évaluation initiale, celles pour lesquelles un PCN a offert ses bons offices et qui sont en cours d'examen et celles pour lesquelles, à l'issue de son évaluation initiale, un PCN aura décidé de ne pas proposer ses bons offices. Dans leurs rapports sur les activités de mise en œuvre dans des circonstances spécifiques, les PCN se conformeront aux principes de transparence et de confidentialité décrits au paragraphe C-4. 


\section{Commentaire sur les lignes directrices de procédure pour le Comité de l'investissement}

43. Les lignes directrices de procédure annexées à la Décision du Conseil comportent également des lignes directrices à l'intention du Comité qui, dans l'exercice de ses responsabilités, devra :

- Exercer ses attributions efficacement et promptement.

- Étudier les demandes d'assistance des PCN.

- Organiser des échanges de vues sur les activités des PCN.

- Ménager la possibilité de demander des conseils à des partenaires ou à des spécialistes internationaux.

44. Le caractère non contraignant des Principes directeurs interdit au Comité d'agir comme un organe judiciaire ou quasi judiciaire. Les conclusions et les communiqués émanant des PCN (autres que les interprétations des Principes directeurs) ne devront pas non plus être remis en question par un renvoi au Comité de l'investissement. La disposition prévoyant que le Comité doit s'abstenir de tirer des conclusions sur le comportement d'entreprises déterminées a été maintenue dans la Décision même.

45. Le Comité de l'investissement étudiera les demandes d'assistance des PCN, y compris lorsqu'il existe un doute quant à l'interprétation des Principes directeurs dans des circonstances particulières. Ce paragraphe reflète le paragraphe $\mathrm{C} 2 \mathrm{c}$ ) des Lignes directrices de procédure annexées à la Décision du Conseil concernant les PCN, aux termes duquel les PCN sont invités à demander l'avis du Comité de l'investissement en cas de doute quant à l'interprétation des Principes directeurs dans ces circonstances.

46. Lors des discussions sur les activités des PCN, le Comité pourra formuler des recommandations, en tant que de besoin, afin d'en améliorer le fonctionnement, notamment dans l'optique d'une mise en œuvre efficace des Principes directeurs.

47. Le Comité de l'investissement examinera également les griefs dûment motivés émanant d'un pays ayant adhéré aux Principes directeurs, d'un organe consultatif ou d'OECD Watch reprochant à un PCN de ne pas s'acquitter de ses obligations procédurales de mise en œuvre des Principes directeurs dans des circonstances spécifiques. Cette 
disposition complète les dispositions des Lignes directrices de procédure concernant l'établissement de rapports d'activité par les PCN.

48. Clarifier la signification des Principes directeurs au niveau multilatéral restera une responsabilité essentielle du Comité de l'investissement, afin de garantir l'homogénéité d'interprétation des Principes directeurs d'un pays à l'autre. Le Comité examinera également les demandes motivées formulées par un pays ayant adhéré aux Principes directeurs, par un organe consultatif ou par OECD Watch, concernant la conformité entre une interprétation des Principes directeurs par un PCN et l'interprétation du Comité.

49. Pour s'engager auprès des pays non adhérents sur des questions couvertes par les Principes directeurs, le Comité pourra inviter des pays non adhérents intéressés à assister à ses réunions, aux Tables rondes annuelles sur la responsabilité des entreprises ou à des réunions consacrées à des projets spécifiques dans le domaine de la conduite responsable des entreprises.

50. Enfin, le Comité peut décider de faire appel à des spécialistes pour examiner des questions plus générales (par exemple le travail des enfants, les droits de l'homme) ou des problèmes précis et rédiger des rapports à ce propos, ou en vue d'améliorer l'efficacité des procédures. A cette fin, le Comité de l'investissement pourra faire appel à des experts de l'OCDE, d'autres organisations internationales, d'organes consultatifs, d'organisations non gouvernementales, de milieux universitaires et autres. Il est entendu que ces experts ne constitueront pas un groupe spécial chargé de régler des problèmes précis. 



\section{ORGANISATION DE COOPÉRATION ET DE DÉVELOPPEMENT ÉCONOMIQUES}

L'OCDE est un forum unique en son genre où les gouvernements œuvrent ensemble pour relever les défis économiques, sociaux et environnementaux que pose la mondialisation. L'OCDE est aussi à l'avant-garde des efforts entrepris pour comprendre les évolutions du monde actuel et les préoccupations qu'elles font naître. Elle aide les gouvernements à faire face à des situations nouvelles en examinant des thèmes tels que le gouvernement d'entreprise, l'économie de l'information et les défis posés par le vieillissement de la population. L'Organisation offre aux gouvernements un cadre leur permettant de comparer leurs expériences en matière de politiques, de chercher des réponses à des problèmes communs, d'identifier les bonnes pratiques et de travailler à la coordination des politiques nationales et internationales.

Les pays membres de l'OCDE sont: l'Allemagne, l'Australie, l'Autriche, la Belgique, le Canada, le Chili, la Corée, le Danemark, l'Espagne, l'Estonie, les États-Unis, la Finlande, la France, la Grèce, la Hongrie, l'Irlande, l'Islande, Israël, l'Italie, le Japon, le Luxembourg, le Mexique, la Norvège, la Nouvelle-Zélande, les Pays-Bas, la Pologne, le Portugal, la République slovaque, la République tchèque, le Royaume-Uni, la Slovénie, la Suède, la Suisse et la Turquie. L'Union européenne participe aux travaux de l'OCDE.

Les Éditions OCDE assurent une large diffusion aux travaux de l'Organisation. Ces derniers comprennent les résultats de l'activité de collecte de statistiques, les travaux de recherche menés sur des questions économiques, sociales et environnementales, ainsi que les conventions, les principes directeurs et les modèles développés par les pays membres. 


\section{Les principes directeurs de l'OCDE à l'intention des entreprises multinationales ÉDITION 2011}

\section{Table des matières}

La Déclaration sur l'investissement international et les entreprises multinationales

Partie I - Les Principes directeurs de l'OCDE à l'intention des entreprises multinationales : Recommandations pour une conduite responsable des entreprises dans le contexte international

\section{Préface}

I. Concepts et principes

II. Principes généraux

III. Publication d'informations

IV. Droits de l'homme

V. Emploi et relations professionnelles

VI. Environnement

VII. Lutte contre la corruption, la sollicitation de pots-de-vin et d'autres formes d'extorsion

VIII. Intérêts des consommateurs

IX. Science et technologie

$\mathrm{X}$. Concurrence

XI. Fiscalité

Partie II - Procédures de mise en œuvre des Principes directeurs de l'OCDE à l'intention des entreprises multinationales

Amendement de la décision du Conseil sur les principes directeurs de l'OCDE à l'intention des entreprises multinationales

Lignes directrices de procédure

Commentaire sur les procédures de mise en œuvre des Principes directeurs de l'OCDE à l'intention des entreprises multinationales

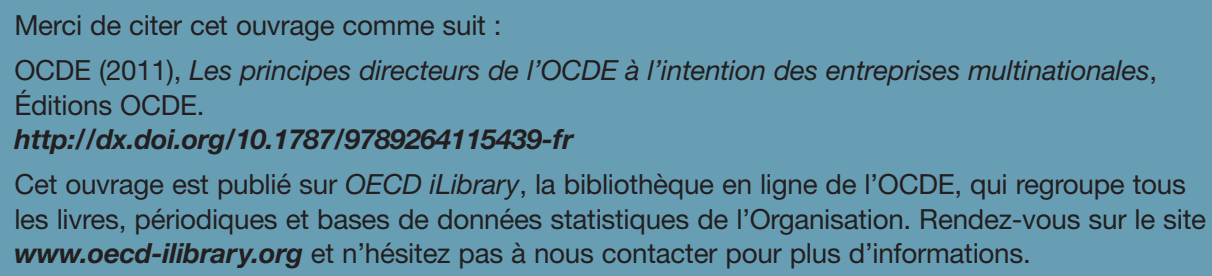

